\begin{tabular}{|c|l|}
\hline Title & A skeleton structure of self-replicating dynamics \\
\hline Author(s) & Nishiura, Y.; Ueyama, D. \\
\hline Citation & Hokkaido University Preprint Series in Mathematics, 396, 1-27 \\
\hline Issue Date & 1997-10-1 \\
\hline DOI & 10.14943/83542 \\
\hline Doc URL & http://hdl.handle.net/2115/69146 \\
\hline Type & bulletin (article) \\
\hline File Information & pre396.pdf \\
\hline
\end{tabular}

Instructions for use 


\title{
A Skeleton Structure of Self-replicating Dynamics
}

\author{
Y. Nishiura and D. Ueyama
}

Series \#396. October 1997 


\section{HOKKAIDO UNIVERSITY PREPRINT SERIES IN MATHEMATICS}

\#371 T. Nakazi and H. Sawada, The commutator ideal in Toeplitz algebras for uniform algebras and the analytic structure, 9 pages. 1997.

\#372 M.-H. Giga and Y. Giga, Stability for evolving graphs by nonlocal weighted curvature, 70 pages. 1997.

\#373 T. Nakazi, Brown-Halmos type theorems of weighted Toeplitz operators, 14 pages. 1997.

\#374 J. Inoue and S.-E. Takahashi, On characterizations of the image of Gelfand transform of commutative Banach algebras, 30 pages. 1997.

\#375 L. Solomon and H. Terao, The double coxeter arrangement, 21 pages. 1997.

\#376 G. Ishikawa and T. Morimoto, Solution surfaces of Monge-Ampère equations, 15 pages. 1997.

\#377 G. Ishikawa, A relative transversality theorem and its applications, 16 pages. 1997.

\#378 J. Inoue and T. Nakazi, On the zeroes of solutions of an extremal problem in $H^{1}, 14$ pages. 1997.

\#379 Y. Giga and K. Ito, On pinching of curves moved by surface diffusion, 12 pages. 1997.

\#380 F. Hiroshima, Weak coupling limit removing an ultraviolet cut-off for a Hamiltonian of particles interacting with a scalar field, 39 pages. 1997.

\#381 Y. Giga, S. Matsui and Y. Shimizu, On estimates in Hardy spaces for the Stokes flow in a half space, 13 pages. 1997.

\#382 A. Arai, A new estimate for the ground state energy of Schrödinger operators, 12 pages. 1997.

\#383 M. Nakamura and T. Ozawa, The Cauchy problem for nonlinear wave equations in the Sobolev space of critical order, 24 pages. 1997.

\#384 K. Ito, Asymptotic stability of planar rarefaction wave for scalar viscous conservation law, 8 pages. 1997.

\#385 A. Arai, Representation-theoretic aspects of two-dimensional quantum systems in singular vector potentials: canonical commutation relations, quantum algebras, and reduction to lattice quantum systems, 32 pages. 1997.

\#386 P. Aviles and Y. Giga, On lower semicontinuity of a defect energy obtained by a singular limit of the Ginzburg-Landau type energy for gradient fields, 21 pages. 1997.

\#387 T. Nakazi and T. Yamamoto, Norms of some singular integral operators and their inverse operators, 28 pages. 1997.

\#388 M.-H. Giga and Y. Giga, Remarks on convergence of evolving graphs by nonlocal curvature, 18 pages. 1997.

\#389 T. Tsukada, Reticular Lagrangian singularities, 41 pages. 1997.

\#390 M. Nakamura and T. Ozawa, The Cauchy problem for nonlinear wave equations in the homogeneous Sobolev space, 12 pages. 1997.

\#391 Y. Giga, M. Ohnuma and M.-H. Sato, On strong maximum principle and large time behaviour of generalized mean curvature flow with the Neumann boundary condition, 24 pages. 1997.

\#392 T. Tsujishita and H. Watanabe, Monoidal closedness of the category of simulations, 24 pages. 1997.

\#393 T. Arase, A remark on the quantale structure of multisets, 10 pages. 1997.

\#394 N. H. Bingham and A. Inoue, Extension of the Drasin-Shea-Jordan theorem, 16 pages. 1997.

\#395 N. H. Bingham and A. Inoue, Ratio Mercerian theorems with applications to Hankel and Fourier transforms, 30 pages. 1997. 


\title{
A Skeleton Structure of Self-replicating Dynamics
}

\author{
Yasumasa Nishiura and Daishin Ueyama \\ Laboratory of Nonlinear Studies and Computations, \\ Research Institute for Electronic Science, \\ Hokkaido University, \\ Kita-ku, Sapporo, 060, JAPAN
}

\begin{abstract}
A skeleton dynamics for the self-replicating patterns (SRP) of reaction diffusion system is presented. Selfreplicating dynamics can be regarded as a transient process from a localized trigger to a stable Turing pattern or oscillatory Turing pattern. It looks like a reverse process of usual coarsening phenomena, i.e., the number of unit localized pattern increases until the domain is filled by them completely. SRP was found in several chemical reaction models, for instance, the Gray-Scott model as well as in real experiments. The most difficult point to describe SRP lies in the fact that it is truly a transient phenomenon in the sense that it can be captured neither as a definite object in dynamical system theory like an attractor nor an orbit itinerating among saddle points in the phase space. To our knowledge, it is not known that what kind of dynamical framework is suitable to clarify the behavior of SRP. The aim is to give a new point of view to describe such a transient dynamics of SRP on a finite interval. Especially we concentrate on the basic mechanism causing SRP from a bifurcational view point by employing a new model system and its finite-dimensional conipartment model which shares common qualitative features with the Gray-Scott model. By a careful anatomy of global bifurcation diagrams, the skeleton dynamics of SRP comes from a hierarchy structure of the subcritical bifurcating loops of oscillatory branches of pulse type. It should be noted that these loops themselves do not constitute the skeleton dynamics of SRP, but the ruins of them do it. In other words, the aftereffect of the hierarchy structure manifests the dynamics of SRP. The most important ingredient of an organizing center from which the whole hierarchy structure of $\mathrm{SRP}$ emerges is Bogdanov-Takens-Turing singularity as well as the existence of stable equilibrium point, which indicates universality of the above structure in the class of nonlinearities sharing this character.
\end{abstract}

Key words: self-replicating pattern, reaction diffusion system, pulse solution, Turing pattern, wave splitting, Bogdanov-Takens point

PACS codes: $68.35 . \mathrm{Fx}, 82.20 . \mathrm{Wt}, 82.40 . \mathrm{Bj}, 82.20 . \mathrm{Mj}$

MSC codes: $34 \mathrm{~A} 47,35 \mathrm{~B} 32,35 \mathrm{~K} 57,35 \mathrm{R} 35,58 \mathrm{~F} 14$ 


\section{Introduction}

Self-replicating patterns have recently been observed numerically in a reaction diffusion system ([1], [2], [5], [4]) as well as in laboratories ([3], [7], [8]). A prototypical model is the irreversible Gray-Scott model that exhibits a variety of new patterns including spots that self replicate and develop into a variety of asymptotic states in two dimensions ([1]) as well as pulses that self replicate in one dimension ([2]). A rigorous analysis concerning the existence of steady single pulse as well as nonexistence of traveling pulses has been done quite recently by [6]. Nevertheless very little is known about the mechanism which drives the replication dynamics itself. The aim of this paper is to present a key mechanism for the self-replicating dynamics with the aid of a new model system that shares qualitatively the same dynamics as in the Gray-Scott model. The self-replicating dynamics depicted as in Fig. 1.1 can be regarded as a transient process from a localized trigger to a stable Turing pattern or oscillatory Turing pattern (i.e., Turing pattern destabilized by Hopf bifurcation).

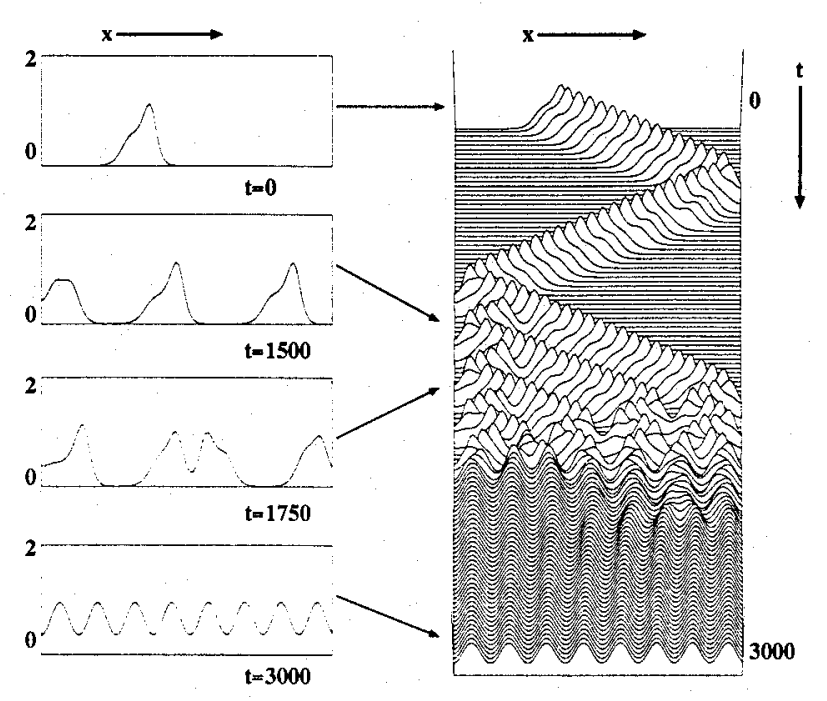

Figure 1.1: Self-replication for PDE model (1) $\left(D_{u}=0.001, D_{v}=0.004, \alpha=0.27169\right.$, width of interval $=4$ ).

It looks like a reverse process of the usual coarsening phenomena, i.e., the number of unit localized pattern increases until the domain is filled by them completely. More precisely, on a finite interval, there are three different phases of dynamics of SRP:

1. Traveling phase: an isolated pulse-like pattern (i.e., away from other pulses and boundary) travels almost without changing its shape.

2. Splitting phase: some of the pulses split into two parts.

3. Convergence to a final state: after several circulation of the above two phases, and the number of localized patterns exceeds a critical level, then the orbit approaches an asymptotic state. 
The goal is to present a hidden structure causing SRP by taking a bifurcational view point and employing a finite-dimensional compartment model which keeps the essential features of our model system. At present it is quite hard for us to trace globally all the time-periodic branches of PDE model without the reduction to the finite-dimensional model. The difficulty lies in the fact that SRP is neither an invariant set like an timeperiodic branch in $X=S \times P$ where $S$ is a solution space and $P$ is a parameter space, nor an itinerary orbit traveling from a steady state of higher instability to that of lower instability along stable (or unstable) manifolds of them like a coarsening process of CahnHilliard or bistable scalar reaction diffusion equations. Nevertheless, by a careful study of bifurcation diagram, it turns out that the skeleton of SRP dynamics stems from a hierarchy structure of the subcritical time-periodic bifurcating branches of multi-pulse type in $X$. Each branch originates in a secondary bifurcation of Hopf type with loop structure from the associated Turing (steady state) branch, namely it bifurcates from a Turing branch and comes back to the same branch. When the size of the loop becomes larger, the type of bifurcation changes from supercritical to subcritical, and hence the loop has a limiting point where stable and unstable time-periodic branches coalesce. Hierarchy structure means that such limiting points are located almost at the same value of the bifurcation parameter like Fig. 3.17 as well as the connection among stable and unstable manifolds explained in Section 3. The simplest case consists of only one limiting point and a Turing branch like Fig. 1.2.

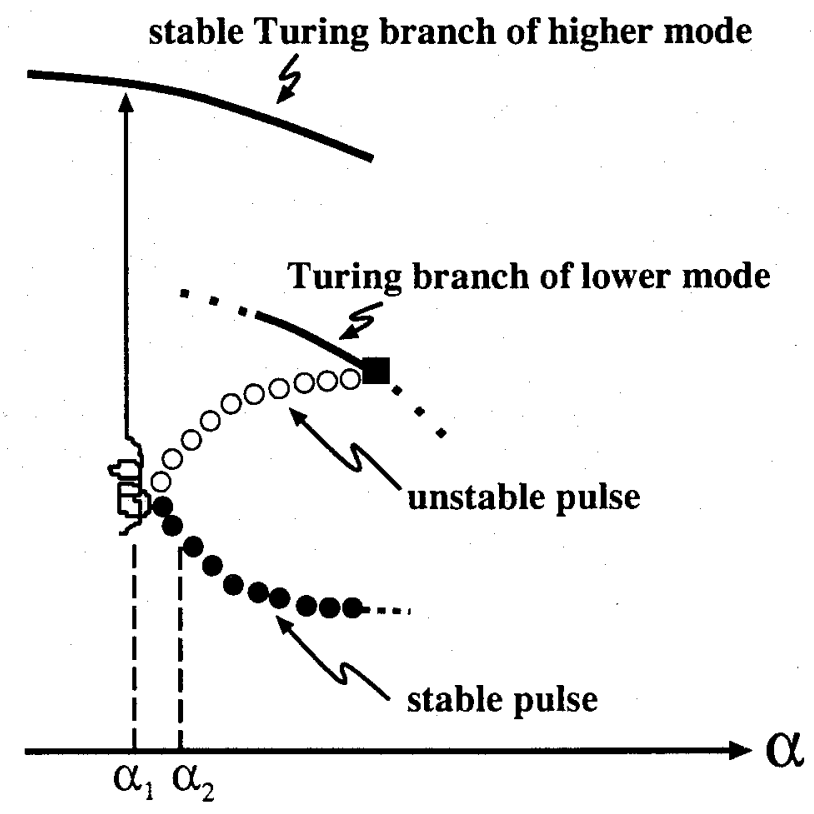

Figure 1.2: A basic mechanism for self-replication.

Suppose one takes a parameter value at $\alpha=\alpha_{1}$ which is right after the limiting point where all time-periodic solutions already disappear there, and starts with an initial data of pulse shape which is close to a snapshot of stable periodic solution at $\alpha=\alpha_{2}$ near the limiting point, it behaves like an time-periodic solitary pulse for a while, and then jumps 
to a Turing branch of higher modes (see Fig. 1.3). The replication occurs exactly when the orbit leaves the area close to the limiting point in the phase space.

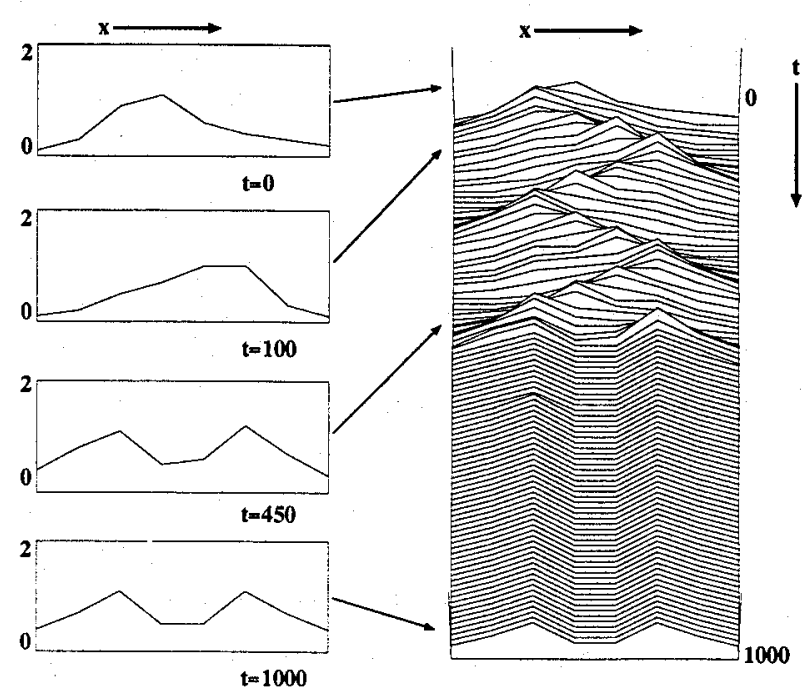

Figure 1.3: Splitting of 1-pulse $(d=0.113, \alpha=0.28, n=8)$ : a pulse-like pattern of the compartment model (3) oscillates temporarily and then splits into two parts.

It should be noted that the orbit only feels the aftereffect of limiting point of timeperiodic solutions of pulse type since there remains no invariant set in the phase space, and this aftereffect corresponds to the dynamical stage where it behaves like an traveling pulse before and after replication. Intuitively this aftereffect reminds us of the intermittency of mapping, and they are, in fact, very close each other in spirit. When the system size becomes larger, the longer the sequence of limiting points, and the splitting process continues until the mode number (or the number of pulses) reaches the level enough to cover the whole region. The most important ingredient of an organizing center from which the whole hierarchy structure of SRP emerges is the Bogdanov-Takens-Turing (BTT) singularity as well as the existence of stable equilibrium point, which indicates universality of the self-replicating phenomenon in the class of nonlinearities sharing this character. It is unclear at present, however, that such a global hierarchy structure can be obtained by local unfolding of BTT point.

\section{The model}

\subsection{PDE model}

We present a minimal model satisfying three conditions: firstly it has a Bogdanov-Takens singularity, secondly the Turing instability occurs at the saddle-node branch of equilibria, and thirdly it has a stable constant state independent of parameters, say $(0,0)$. These features seem to be common among the nonlinearities which give rise to SRP such as 
the Gray-Scott model. The following model system, which seems simpler than the GrayScott model, can serve as one of the prototype models to investigate the self-replicating dynamics:

$$
\left\{\begin{array}{l}
\frac{\partial u}{\partial t}=D_{u} \nabla^{2} u+u\left(u-v^{2}-\alpha\right) \\
\frac{\partial v}{\partial t}=D_{v} \nabla^{2} v+k u-v,
\end{array}\right.
$$

where $D_{u}$ and $D_{v}$ are diffusion coefficients, $\alpha$ and $k$ are non-negative parameters. We mainly consider (1) on a finite interval subject to Neumann (zero flux) boundary conditions. As we will see, this model system and the Gray-Scott model share a remarkably similar qualitative behavior. The corresponding ODE kinetics is

$$
\left\{\begin{array}{l}
\frac{d u}{d t}=u\left(u-v^{2}-\alpha\right) \\
\frac{d v}{d t}=k u-v
\end{array}\right.
$$

The two parameters $\alpha$ and $k$ control the ODE dynamics. The nullclines and the typical flows are drawn in Fig. 2.1 and Fig. 2.2. When $\alpha$ is small, (2) has two nontrivial constant states; the upper one is a stable focus and the lower one is a saddle as in Fig. 2.1. As $\alpha$ becomes larger, through saddle-node bifurcation, (2) becomes monostable like Fig. 2.2.

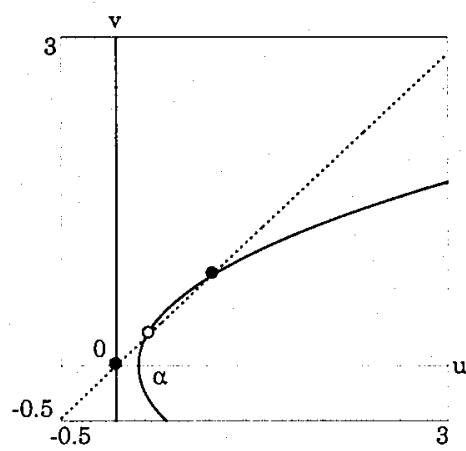

(a)

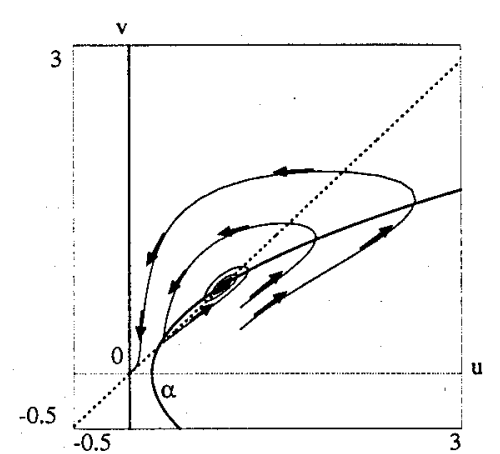

(b)

Figure 2.1: ODE kinetics (bistable)

A codimension 2 singularity called Bogdanov-Takens point (BT-point) appears when both $\alpha$ and $k$ vary. Fig. 2.3 shows how the dynamics changes around the BT-point. When $k$ is fixed to be 0.95 , the bifurcation diagram of (2) with respect to $\alpha$ is depicted in Fig. 2.4, which is a slice of the unfolding of BT-point. Here $\alpha_{c}$ is the saddle-node point and $H_{0}$ is the Hopf bifurcation point. We use the symbols of Table 1 for bifurcation points and branches. 


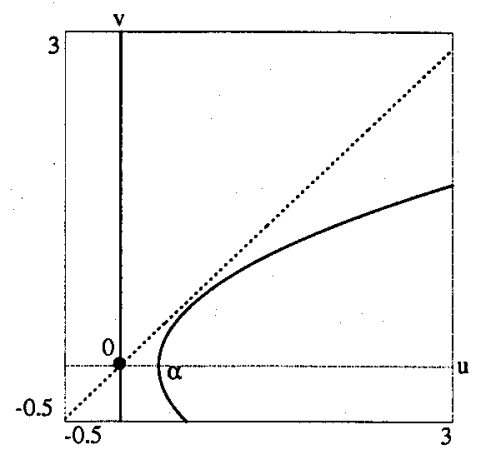

(a)

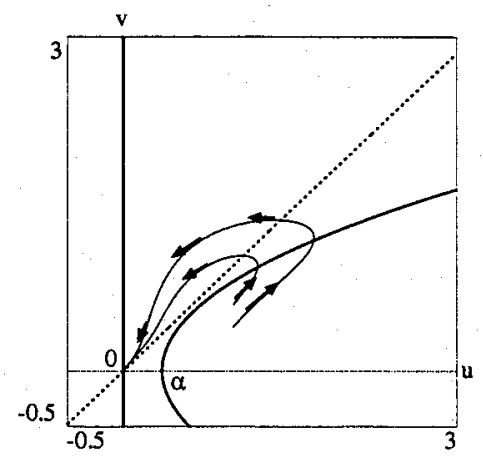

(b)

Figure 2.2: ODE kinetics (monostable)

$\mathbf{k}_{2}^{2}$
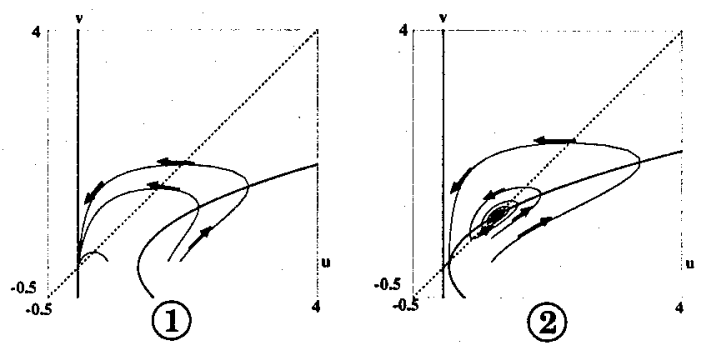

(1)

(2)

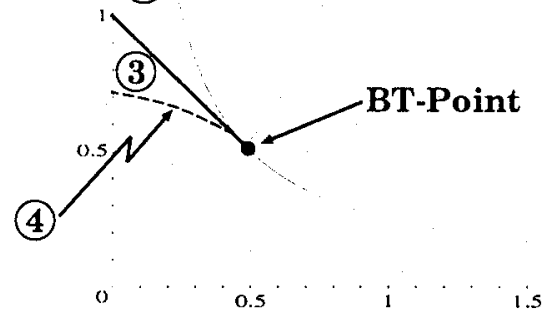

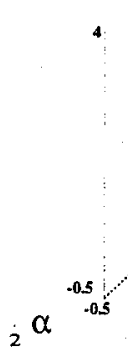

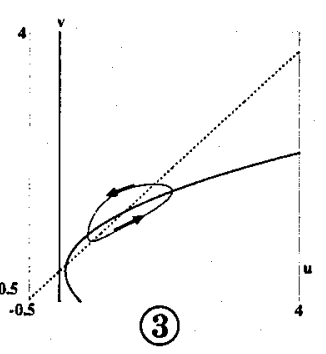

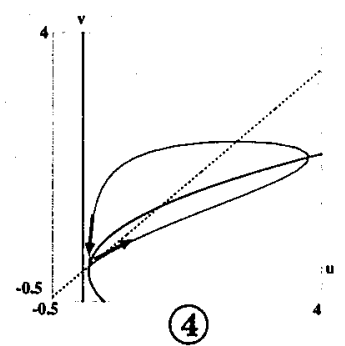

Figure 2.3: Unfolding of Bogdanov-Takens point. 


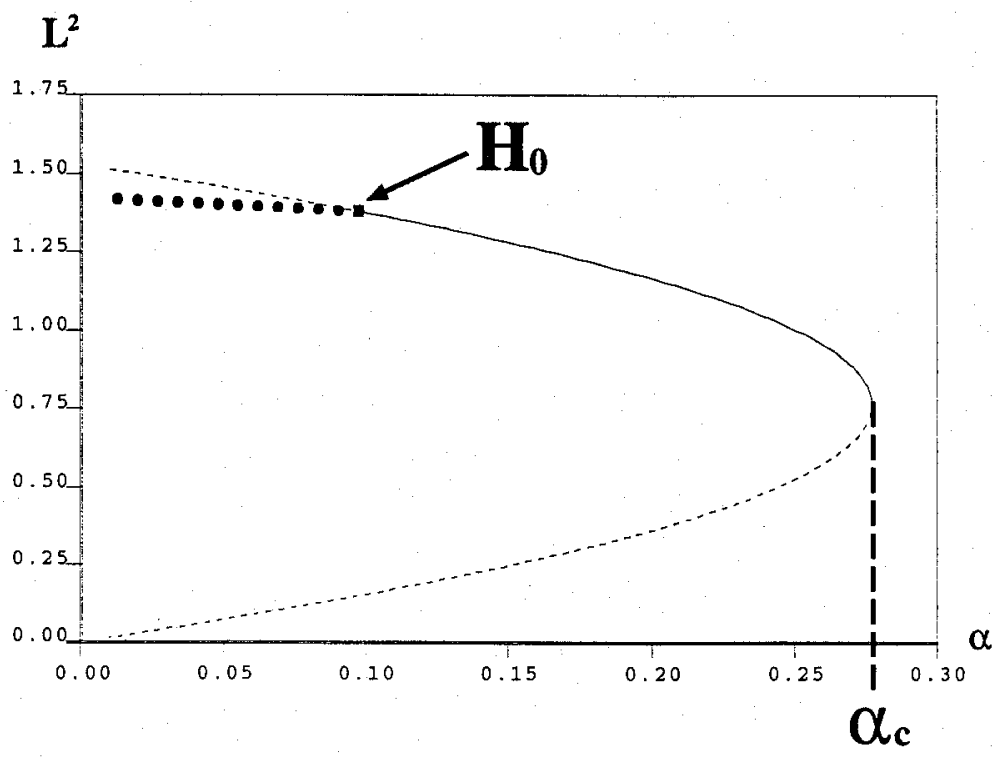

Figure 2.4: Unfolding of BT-point at $k=0.95$.

Table 1: Notation for bifurcation diagrams. 


\subsection{Compartment model}

Although the model system (1) is simply-looking, it is not an easy task to make a numerical tracing of time-periodic branches globally in parameter space. Hence we need a simplified model of (1) which is of finite-dimensional and suitable for numerical tracing. We propose the following diffusionally coupled compartment model where each compartment has the same dynamics as (2):

$$
\left\{\begin{array}{l}
\frac{d u_{i}}{d t}=u_{i}\left(u_{i}-v_{i}^{2}-\alpha\right)+d\left(u_{i-1}-2 u_{i}+u_{i+1}\right) \\
\frac{d v_{i}}{d t}=k u_{i}-v_{i}+d \beta\left(v_{i-1}-2 v_{i}+v_{i+1}\right)
\end{array} i=1, \ldots, n\right.
$$

subject to Neumann boundary conditions on both ends, where $\beta$ is the ratio of diffusivities of $u$ and $v$, and $d$ controls the strength of diffusion. This is a nonlinear ODE system of $2 n$-dimension which is much easier to handle for computation, in particular whole timeperiodic branches can be traced numerically within reasonable time. Moreover, if we take an appropriate number of compartments, the qualitative features of the dynamics and the bifurcation diagrams are the same as the original PDE model 1, which will be discussed in Section 4 . In the following sections, $\beta$ is fixed to be 4 , and the number of compartments $n$ is either $8,16,32$, or 68 .

\section{Self-replication of pulse type}

Most of the exotic dynamics occur near the saddle-node branch in Fig. 2.4. There are at least two types of SRP dynamics: one is SRP of pulse type where pulse-like waves selfreplicate as in Fig. 1.1, and the other is SRP of front type only observed in bistable region. The latter is based on the existence of the traveling front connecting stable equilibrium to trivial state (0.0), and if the Turing instability occurs to the nontrivial constant state, then we have a SRP of front type when the velocity of the front is appropriate (more precise analysis will be reported elsewhere). On the other hand, SRP of pulse type seems to be more subtle, since there is no apparent clue to the mechanism causing SRP. In this section we try to find a sort of hidden organizing center of SRP of pulse type by a careful study of bifurcation diagrams. Although a lot of steady and time-periodic patterns are found by numerical tracing of bifurcating branches (we used AUTO [9]), those themselves do not constitute the skeleton of the dynamics of SRP like saddle points and their connecting orbits in the description of the coarsening process of binary alloy. However this does not mean that those invariant sets are useless to understand SRP. On the contrary, they play the key role behind the scenes to describe the transient dynamics in the form of aftereffect. The aim of this section is to clarify the meaning of aftereffect in the context of transient dynamics.

\subsection{Bogdanov-Takens-Turing point}

There are three parameters $k, \alpha$, and diffusivity $d$. In what follows we fix the value of $k$ as $k=0.95$ and the ratio of diffusivities as $\beta=D_{v} / D_{u}=4$. The ODE kinetics employed 
in the model system (1) has a Bogdanov-Takens point when $k=1 / \sqrt{2}$ and $\alpha=1 / 2$. Saddle-node and Hopf singularities coalesce at this point in the parameter space. It should be noted that the trivial state $(0,0)$ always exists and is locally asymptotically stable. Therefore the kinetics is of bistable type when $\alpha<\alpha_{c}$, and mono-stable when $\alpha>\alpha_{c}$, where $\alpha_{c}$ is the $\alpha$-value of the saddle-node point. When the diffusion effect is taken into account, we also have the diffusion-driven (Turing) instability at an equilibrium point on the saddle-node parabolic branch in Fig. 2.4. The Turing bifurcation points can be easily computed like Fig. 3.1. Note that in most cases, for a fixed $d$, the highest modenumber of branch primarily bifurcates from the upper branch with respect to $\alpha$. The emanating branch remains as a stable steady state for larger $\alpha$ and become a candidate of final states after splitting. Combining BT-point and Turing instability, we have a highly degenerate singularity, what we call, the Bogdanov-Takens-Turing point by tuning three parameters, which is at least of codimension 3. Since it is quite complicated and tough to study the unfolding of this singularity, we focus on a special section of this singularity by fixing $k=0.95$ and $\beta=D_{v} / D_{u}=4$ as mentioned earlier. We draw the bifurcation diagrams in $\alpha$ vs solution space for a given $d$, and trace the behavior of bifurcating branches when the diffusivity $d$ decreases. For large $d$, say $d=2.5$, the bifurcation diagram is the same as that of the ODE kinetics (see Fig. 2.4), i.e., there appear saddle-node bifurcation of equilibria and the Hopf bifurcation on the upper branch which ends up with the homoclinic orbit. When $d$ decreases, there appears a Turing loop of 1-mode type connecting the upper branch to the lower branch of equilibria via diffusiondriven instability (see Fig. 3.2). The Turing branch in Fig. 3.2 does not look like a loop, since the norm of the ordinate is $L^{2}$. If we switch to a different norm which distinguishes a solution from its reflection at the center of the interval, the loop shape becomes visible like Fig. 3.10. Here "1-mode type" means the Fourier-mode number of the associated eigenfunction at the bifurcation point. This type of Turing bifurcation successively occurs as well as secondary and tertiary bifurcations as $d$ decreases, and the resulting branches form a complicated structure in total as in the following subsections, however it turns out that there is a similarity structure among those branches which enables us to predict what will happen as $d$ becomes small.

\subsection{Overhang of Turing loops and emergence of Hopf barrels}

The Turing branch always overhangs saddle-node branch like Fig. 3.2 and therefore Turing patterns persist over the parameter region where there are no nontrivial constant states (i.e., only zero solution survives there, namely mono-stable region). When the Turing bifurcation point on the upper branch come close to the limiting point of equilibria $\alpha_{c}$ (see Fig. 3.1 (a)) as diffusion $d$ decreases, there appears a "Hopf barrel" as a secondary branch near the Turing bifurcation point like Fig. 3.3.

This Hopf instability is different from the one appeared at $H_{0}$ in Fig. 2.4, in fact, a new pair of complex eigenvalue pops up on the way to the bifurcation point $H_{1}$ of Hopf barrel (see Fig. 3.3 and 3.4) and the associated eigenfunction resembles the spacial derivative of the steady Turing pattern there. This implies that the resulting oscillatory pattern rolls from side to side, not up and down (See Fig. 3.5).

Suppose we have a similar type of Hopf bifurcation at the Turing branch of 2 -mode, we 


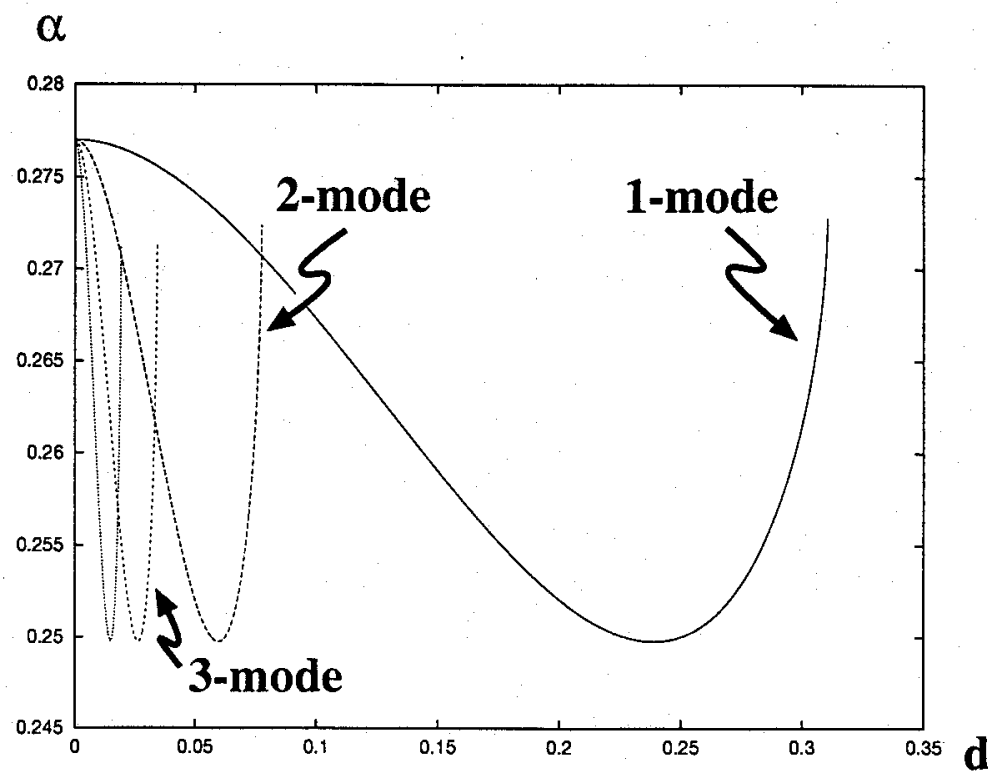

(a)

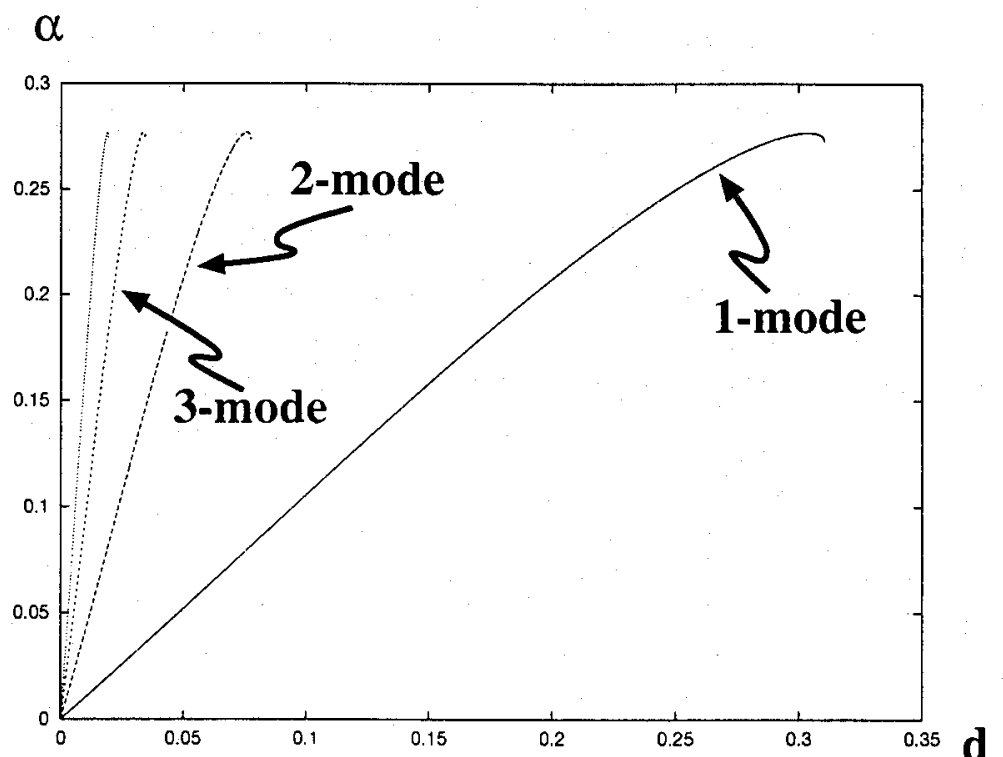

(b)

Figure 3.1: Turing bifurcation curves in $(d, \alpha)$-space $(n=1,2, \ldots, 4)$ for PDE model on $(0, \pi)$ with $k=0.95$; (a) (resp.(b)) shows the location of bifurcation points on the upper (resp. lower) branch of the saddle-node branch. Note that the bifurcation points on the upper branch are concentrated near the saddle-node point $\alpha_{c}=0.277$ (the difference between the maximum and the minimum values of 1 -mode is about 0.026 ), and the smaller $d$, the larger mode-number primarily emerges as $\alpha$ increases. 


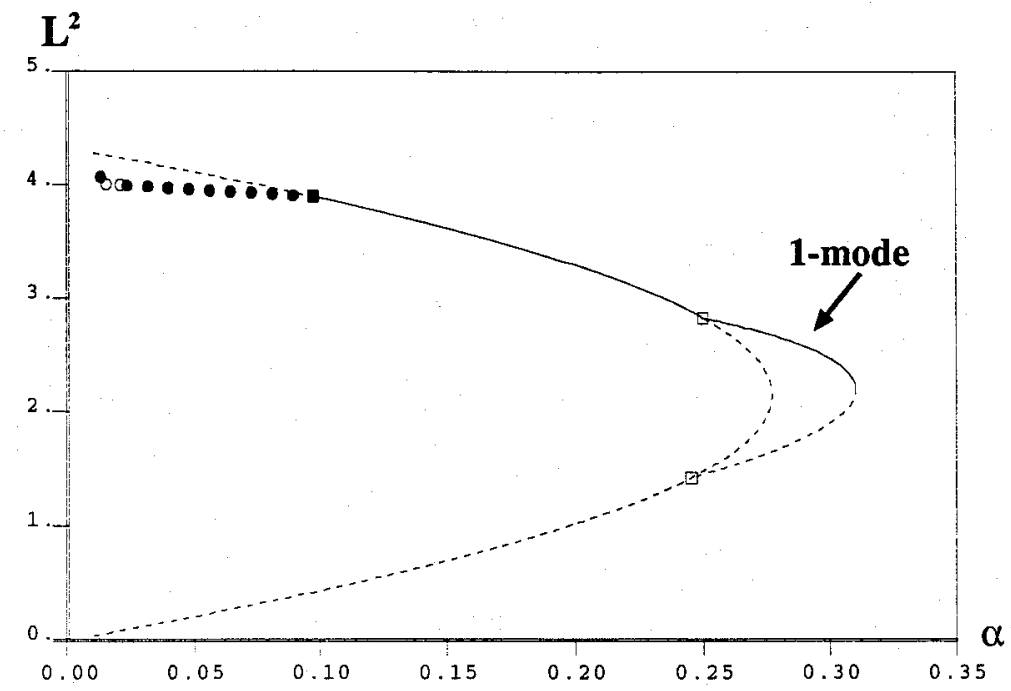

Figure 3.2: Overhang of Turing branch $(d=1.6, n=8)$.
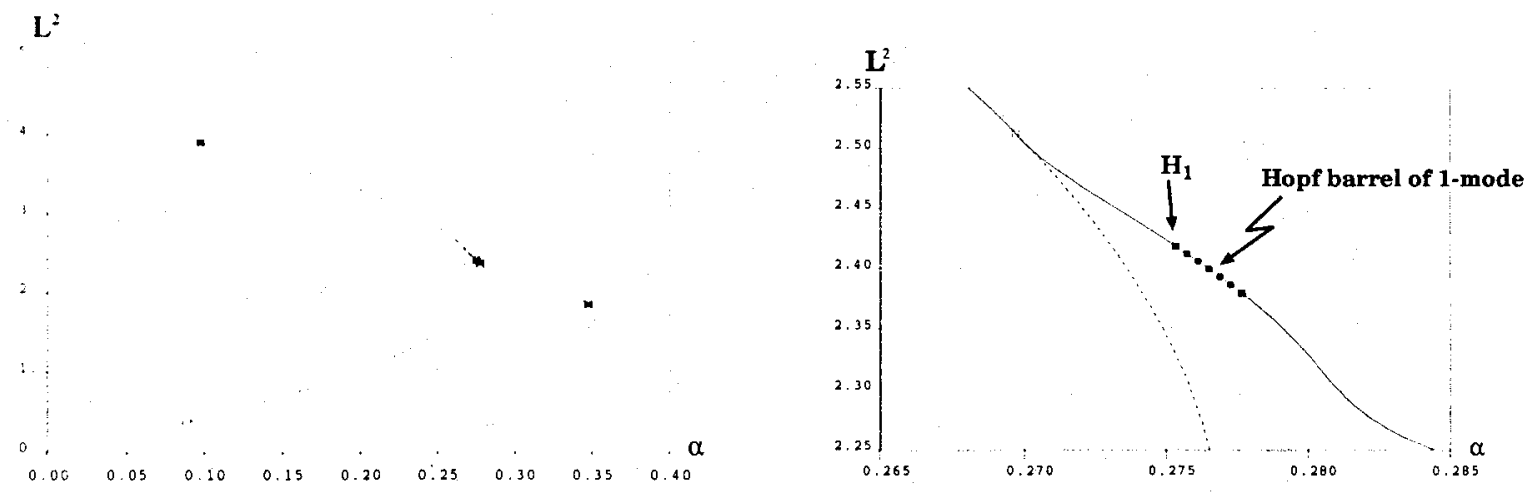

Figure 3.3: Hopf barrel $(d=0.555, n=8)$; the right diagram is a magnification of the left one. The Hopf branch emanates at $H_{1}$ and comes back to the 1-mode branch. 


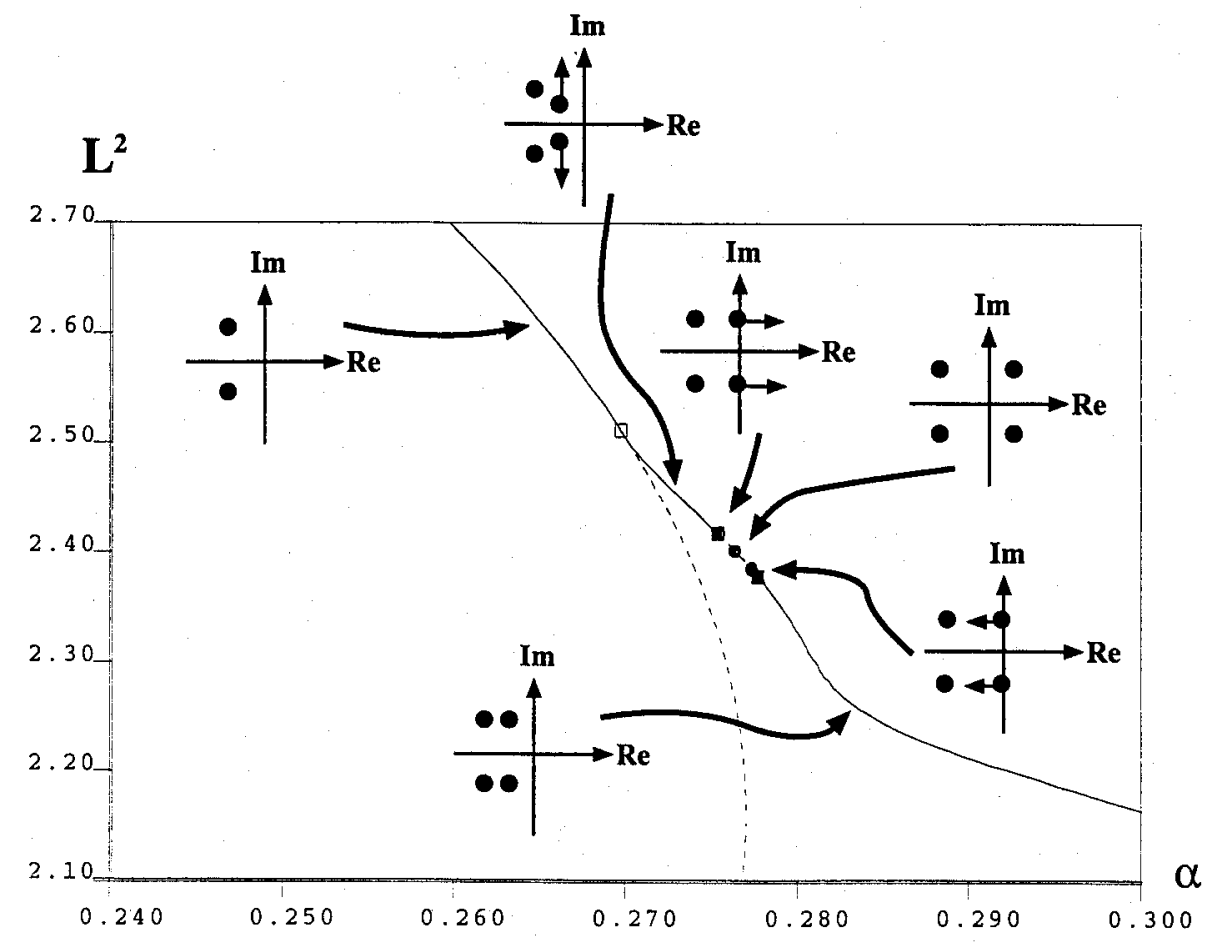

Figure 3.4: Behavior of eigenvalues near the Hopf barrel $(d=0.555, n=8)$; Each spectral distribution is computed at the steady state indicated by arrow.

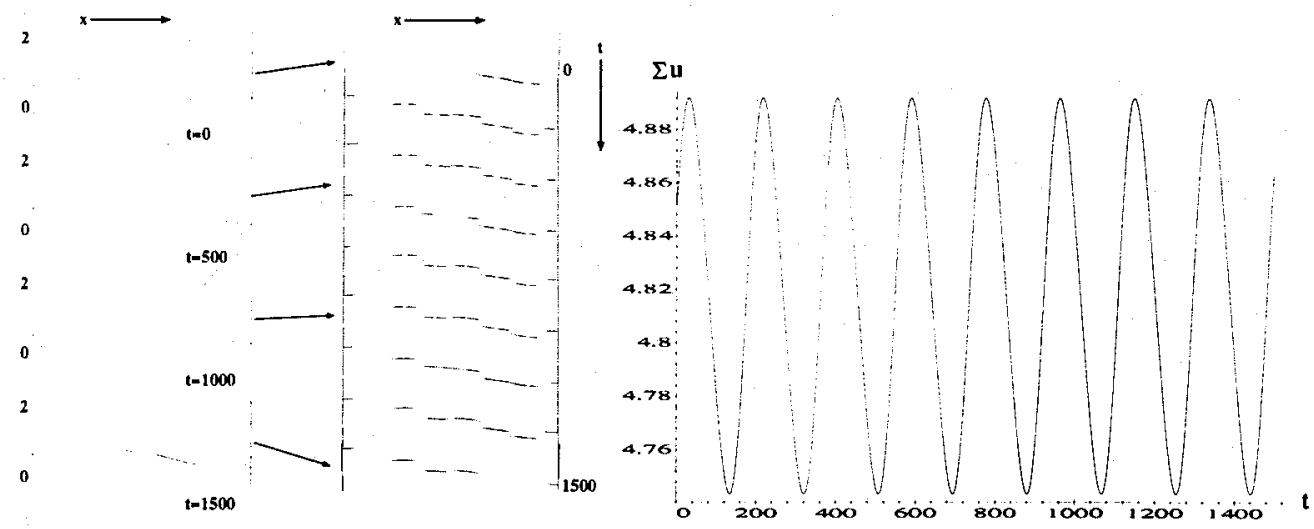

Figure 3.5: Stable oscillatory motion of 1 -mode $(d=0.555, \alpha=0.277, n=8)$. 

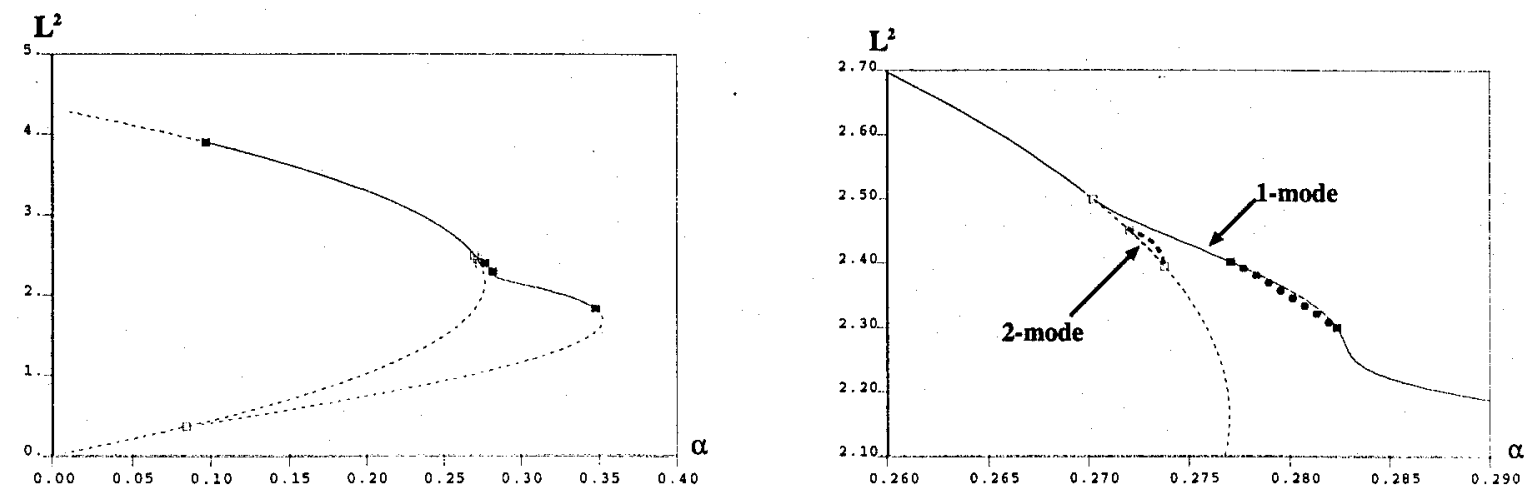

Figure 3.6: Emergence of Turing branch of 2-mode $(d=0.53, n=8)$.
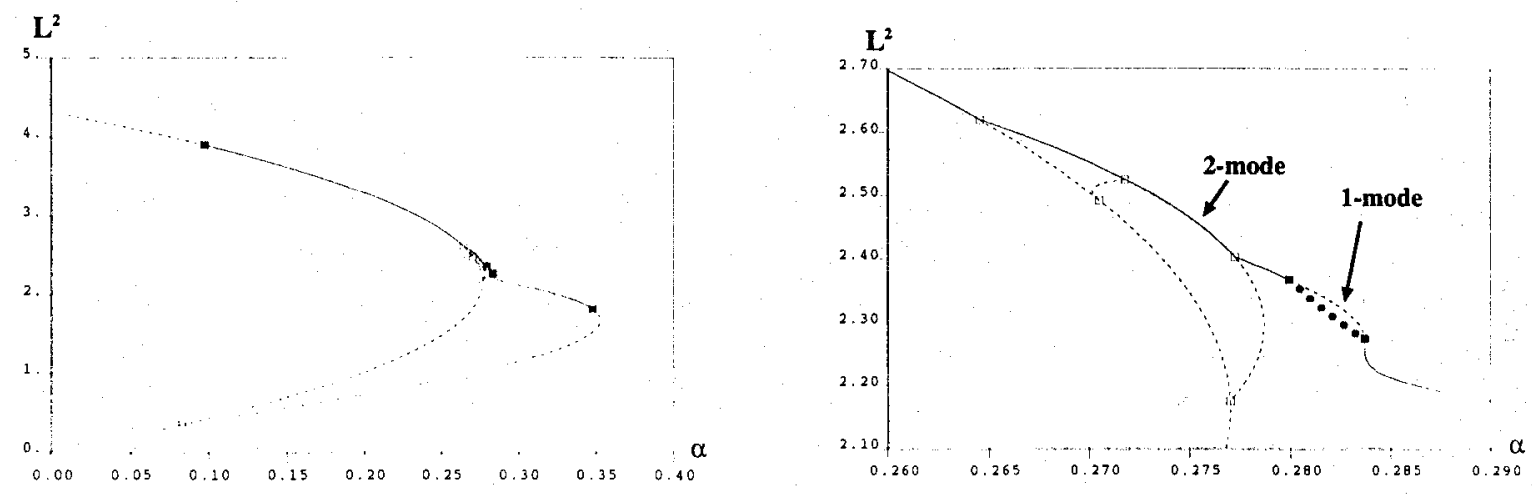

Figure 3.7: Interaction of Turing branches $(d=0.52, n=8)$.
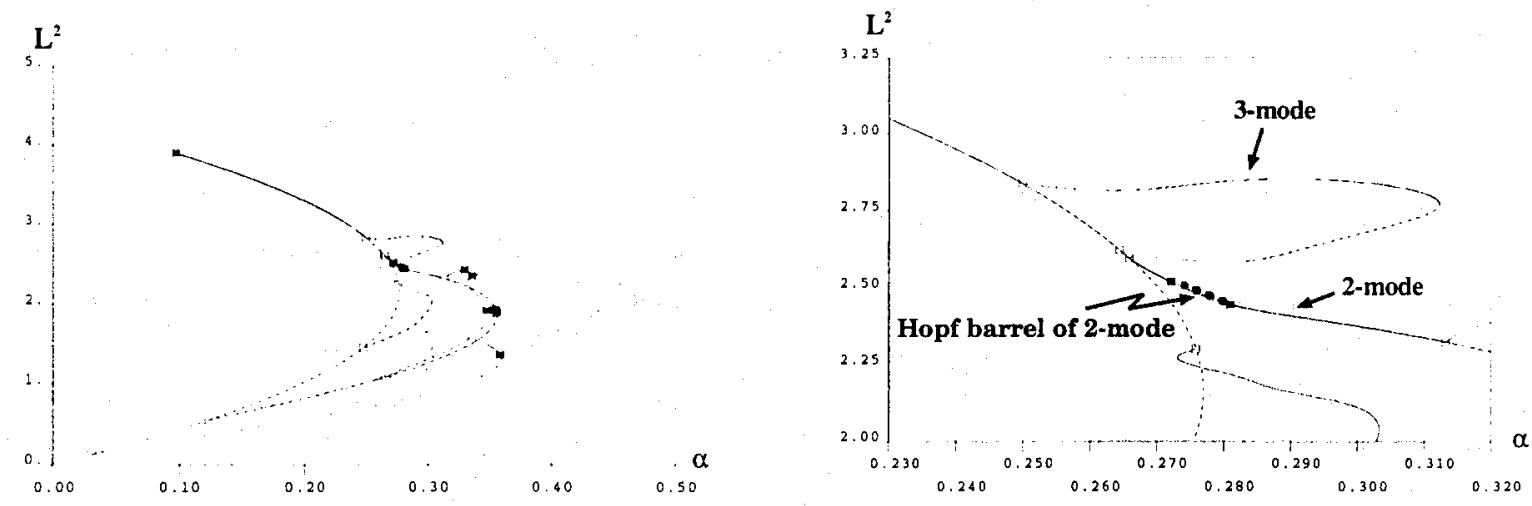

Figure 3.8: Emergence of Hopf barrel on Turing branch of 2-mode $(d=0.2, n=8)$. 


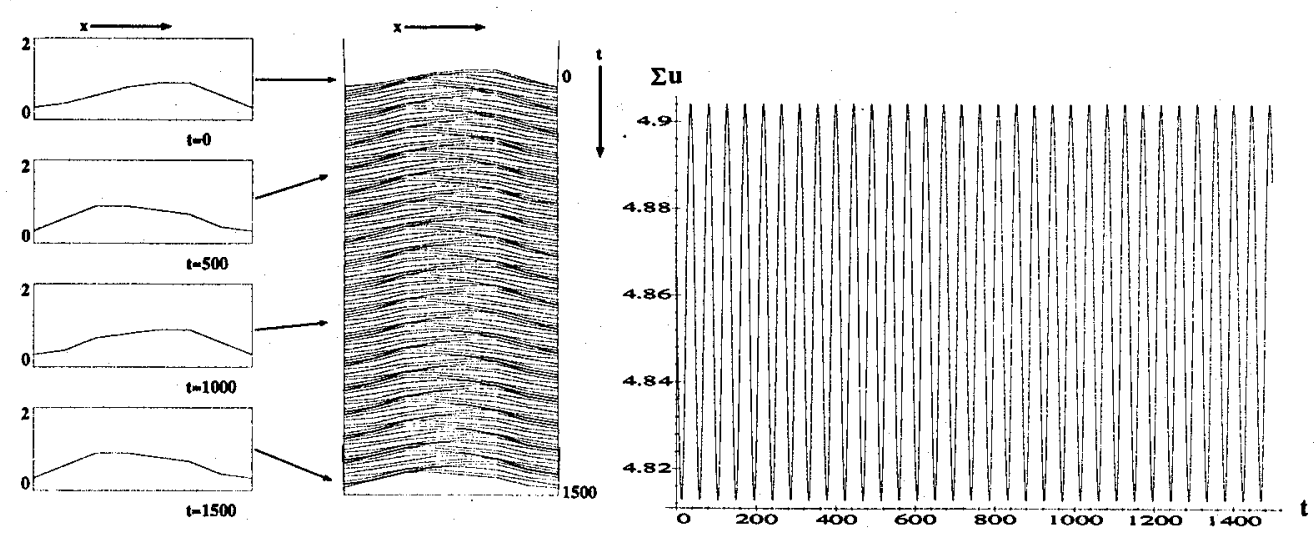

Figure 3.9: Stable 1-pulse $(d=0.2, \alpha=0.275, n=8)$.

expect the existence of time-periodic solution of single pulse shape rolling from side to side. In fact this is the case for our model depicted as in Fig. 3.9, and we discuss about the Hopf barrel on 2-mode branch in the next subsection. In what follows we call such a pulse-like time-periodic solution the oscillatory pulse for simplicity. The sequence of Hopf barrels emanating from even-mode Turing branches is responsible for the coexistence of stable oscillatory multi-pulse solutions, and constitutes an important part of replicating dynamics. The expression "important part of replicating dynamics" may be somewhat misleading, since it will turn out later that the unstable oscillatory multi-pulse solutions also must be taken into account (in fact, once the orbit is trapped in the basin of such a stable branch, it never splits after that), and that the aftereffect of saddle-node bifurcation of these two stable and unstable branches plays a crucial role to explain the transient aspect of replicating dynamics. Namely the self-replicating dynamics can be visible only after all stable oscillatory multi-pulse solutions disappear via saddle-node bifurcation. We will discuss more about this in the following subsections.

\subsection{Interaction of Turing branches and deformation of Hopf bar- rels}

For an appropriate small $d$, the Turing branch of second mode appears as in Fig. 3.6, which interacts with one-mode branch as $d$ decreases through double-zero singularity (codim 2-bifurcation) and the Hopf barrel of one-mode persists as a tertiary bifurcating branch (see Fig. 3.7). As $d$ decreases, the Turing branch of 3-mode appears on the upper side of 2-mode branch, and then Hopf barrel appears on the two-mode branch like Fig. 3.8 , which is of supercritical type. The resulting oscillatory pattern is of pulse type rolling from side to side as in Fig. 3.9. We will see in subsection 3.5 that this oscillatory solution deserves to be called a "pulse" as the system size becomes larger, namely both front and back sides decay quickly to zero. The size of this barrel becomes larger as $d$ decreases, and at the same time, the barrel changes its form from the supercritical type to the 


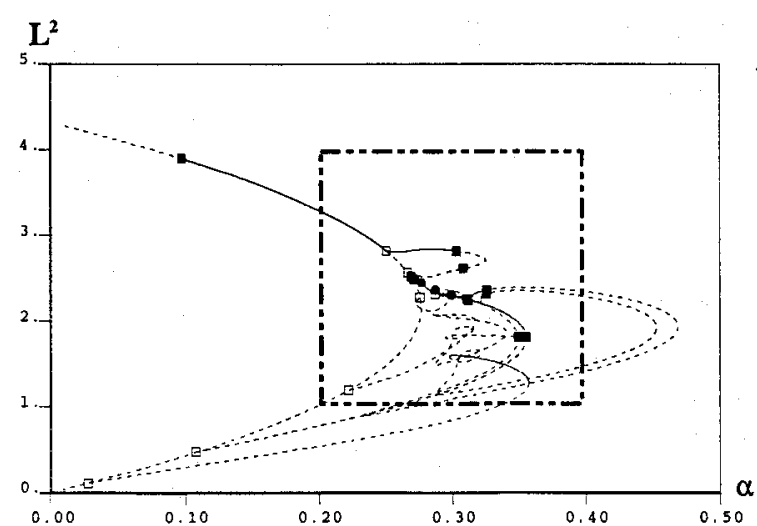

(a-1)

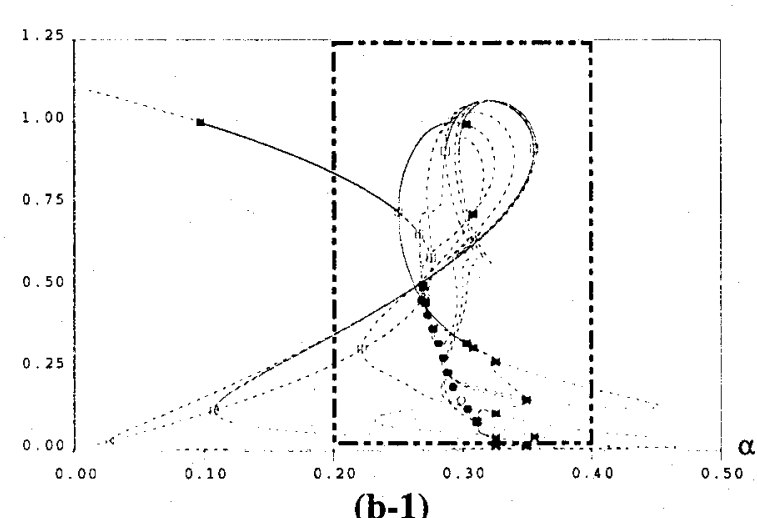

(b-1)

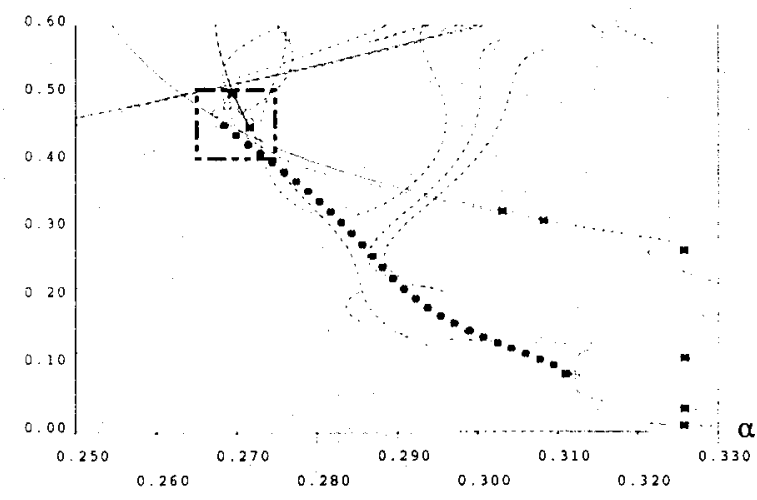

(b-3)

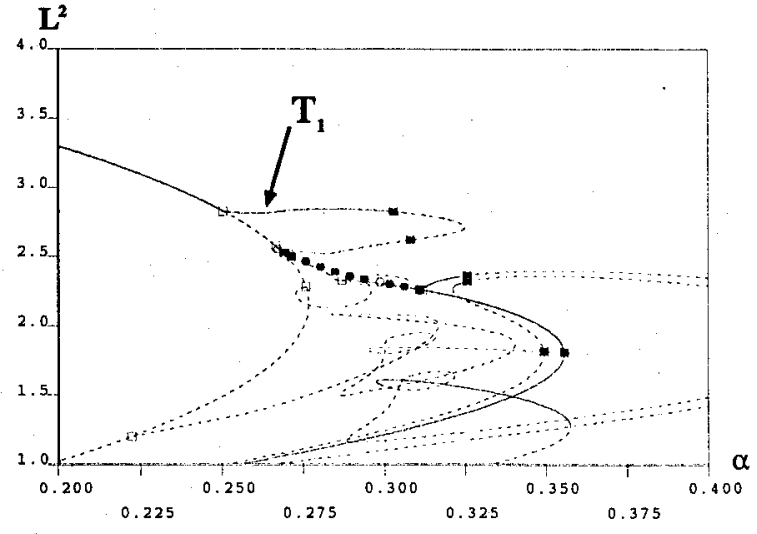

(a-2)
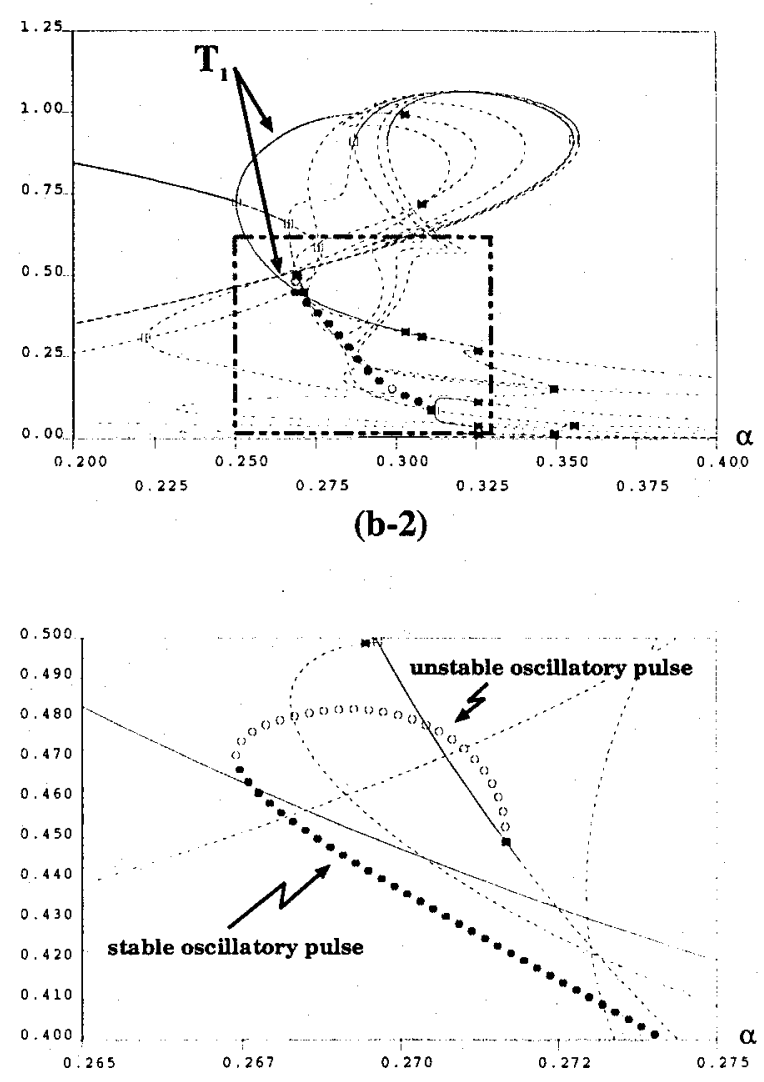

(b-4)

Figure 3.10: Subcriticality and the limiting point of Hopf loop $(d=0.175, n=8)$; The norm of (a)(resp.(b)) is $L^{2}$ (resp.the modulus of the value at the left boundary grid). (a-2) is the magnification of the broken dot square in (a-1), and $(b-i+1)$ is the magnification of the broken dot square in (b-i) for $i=1,2,3$. 


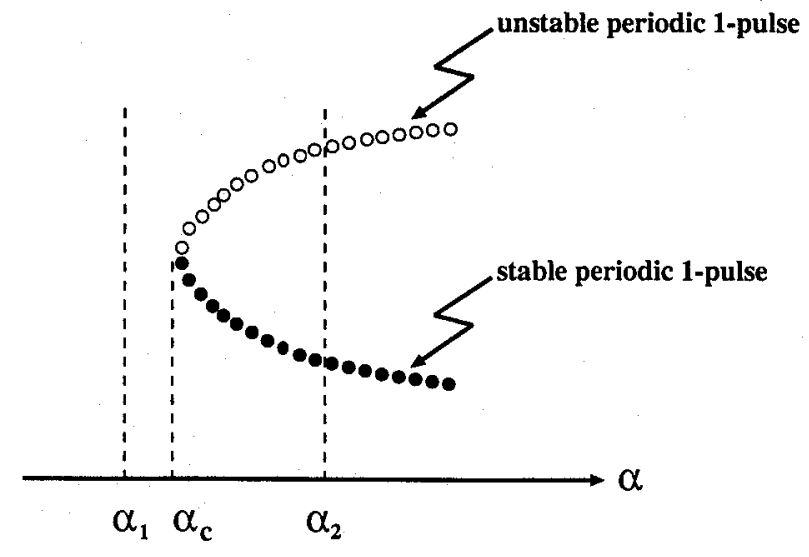

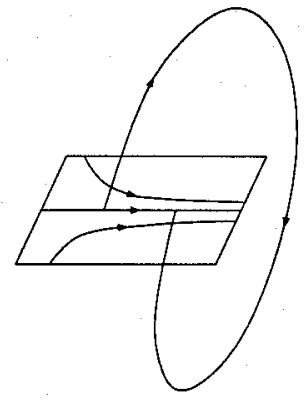

$\alpha_{1}$

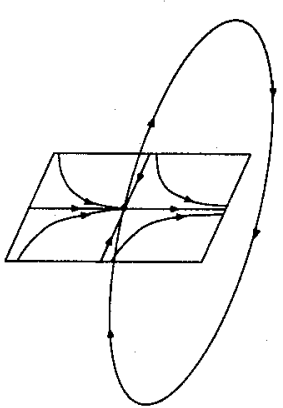

$\alpha_{c}$

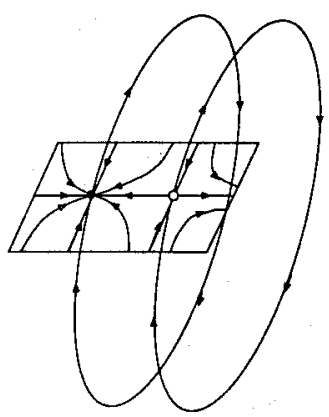

$\alpha_{2}$

Figure 3.11: Dynamics near the limiting point; Even after the event of the saddle-node bifurcation, there still remains an oscillatory motion at $\alpha=\alpha_{1}$. The temporary oscillatory motion lasts longer and longer, when $\alpha_{1}$ approaches $\alpha_{c}$. 
subcritical one. In fact, when $d=0.2$, the whole part of the Hopf branch is represented by black circle (i.e., it consists of only stable branch), and hence no turn around as in Fig. 3.8 , however as $d$ becomes small, the Hopf branch starts to bend and take the form like Fig. $3.10(\mathrm{~b}-4)$ at $d=0.175$. Although this deformation seems innocent from bifurcational point of view, it has a strong influence on the dynamics as discussed below.

\subsection{Fundamental mechanism of self-replicating dynamics}

The change from the supercritical type to the subcritical one has a crucial meaning for the creation of the duplicating dynamics. Subcriticality of Hopf bifurcation gives rise to the existence of limiting point of the oscillatory branch at $\alpha_{c}=0.267407$ (Fig. 3.10(b-4)). It may be instructive to recall that what kind of dynamics occur near the limiting point. The schematic picture Fig. 3.11 shows the three different dynamics when $\alpha$ varies. When $\alpha=\alpha_{2}$, there are stable and unstable time-periodic solutions which coalesce at $\alpha=\alpha_{c}$ and disappear after that, however, even at $\alpha=\alpha_{1}$, there still remains an temporary oscillatory motion as an aftereffect of the limiting point. An exactly the same thing happens near the limiting point of Fig. 3.10(b-4). Fig. 3.12 shows the evolution where $\alpha$ is fixed to be slightly smaller than $\alpha_{c}$ and the initial function is taken from the snapshot of the stable oscillatory solution at $\alpha=\alpha_{2}$. Note that this temporary oscillatory motion keeps running as long as we want, if we choose the parameter $\alpha$ and initial data appropriately. In fact, if $\alpha$ is taken to be much closer to $\alpha_{c}$, then it behaves like an oscillatory pulse ten times longer than before (see Fig. 3.13 and note the difference of time-scale). In other words, although nothing remains after saddle-node bifurcation in the phase space, there is a strong secondary effect due to the coalescence of large-amplitude oscillatory motions when $\alpha\left(<\alpha_{c}\right)$ is close to $\alpha_{c}$.

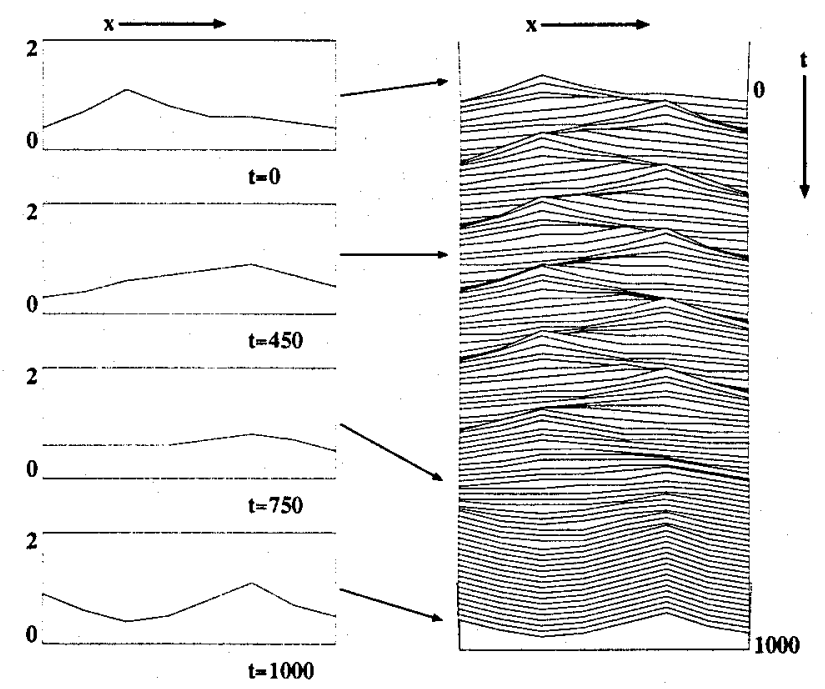

Figure 3.12: From 1-pulse to Turing pattern of 3 -mode $(d=0.175, \alpha=0.2673, n=8)$. 


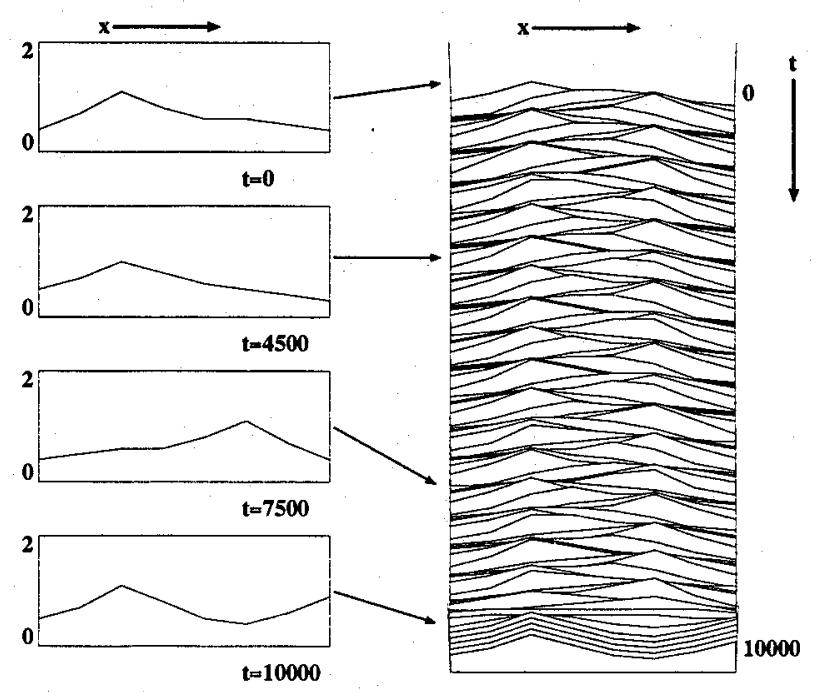

Figure 3.13: From 1-pulse to Turing pattern of 3 -mode $(d=0.175, \alpha=0.2674, n=8)$.

Namely it behaves like an oscillatory pulse for a while, but oscillatory branches already disappear due to the saddle-node bifurcation, it eventually escapes there and then approach the Turing pattern of 3 -mode which corresponds to the stable steady solution $T_{1}$ in Fig. $3.10(a-2),(b-2)$. It is numerically confirmed that the unstable oscillatory pulse (white circle of Fig. $3.10(\mathrm{~b}-4)$ ) has only one unstable Floquet exponent, and that the associated unstable manifold is connected to the 3 -mode Turing pattern $T_{\mathbf{1}}$ and the stable oscillatory pulse (black circle) respectively, hence, by continuation with respect to $\alpha$, we see that it is not a coincidence, but a necessity that the orbit goes to $T_{1}$ after splitting. The important point is that there are two stages of the dynamics, firstly temporal oscillation of pulse type due to the aftereffect of the saddle-node bifurcation, secondly jumping to the Turing branch of higher mode inherited from the connection of the unstable manifold to $T_{1}$. Contrary, without subcriticality (hence no limiting point), there are no exotic behaviors and aftereffects near Hopf barrel. In fact, the Hopf barrel is of supercritical type at $d=0.2$ like Fig. 3.8, then the orbit starting from a 1-pulse shape settles down to a Turing solution of 2 -mode in a damped oscillatory manner like Fig. 3.14 even if $\alpha$ is taken to be close to the onset of Hopf barrel. When $d$ becomes smaller $(d=0.113)$, a Turing pattern of higher mode ( 4 mode) emerges, and the orbit behaves like Fig. 1.3. Namely it oscillates like a one-pulse for a while, then goes to the stable Turing branch of 4 -mode (in fact, it is a mixed solution with 2 -mode).

\subsection{A hierarchy structure of limiting points of oscillatory pulses forms the skeleton of self-replicating dynamics}

When diffusion becomes weak, higher modes of Turing patterns bifurcate from the saddlenode branch and interact with lower modes of Turing patterns, and the resulting bifurcation diagram becomes more and more complicated as $d$ decreases. We extract a hidden 

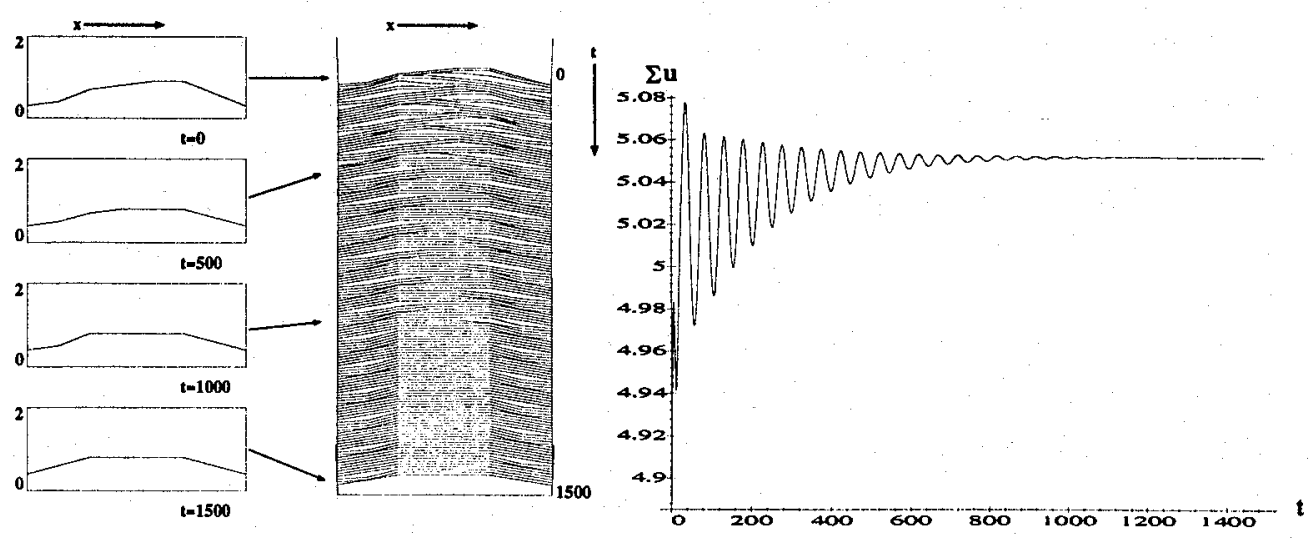

Figure 3.14: Damped oscillation to Turing pattern of 2 -mode $(d=0.2, \alpha=0.27, n=8)$.

structure driving the self-replicating dynamics by careful anatomy of those diagrams. Since our model consists of finitely many compartments (we take $n=32$ in this subsection), there is an upper limit for the mode-number of Turing pattern. However, up to some level, the following hierarchy structure is formed, which is responsible for the replicating dynamics.

1. The stable Hopf barrel from Turing branch of $2 m$-mode appears, swells, and becomes subcritical as $d$ decreases, and hence it has a limiting point at some value of $\alpha$. The corresponding stable oscillatory solution looks like a traveling $m$-pulse bouncing at boundary points (see Fig. 3.15 for $m=2$ ).

2. The existence of such a limiting point persists even after the Hopf barrel deforms, and there appear such limiting points up to some $m_{0}$ in sequence when $d$ decreases. Moreover those points are located almost at the same values of $\alpha$, say $\alpha^{*}$. In fact, for 32-compartment model with $d=0.3$, it is confirmed numerically that at least three different stable oscillatory pulses (from 1-pulse to 3-pulse) exist on each solid interval as in Fig. 3.16, and they cease to exist around $\alpha^{*}=0.27$. This strongly suggests that the bifurcation diagram of Fig. 3.17(b) is plausible; each oscillatory branch has a limiting point and they make an almost straight line. This remarkable coincidence of location of limiting points is a key ingredient to explain the transitory aspect of self-replication dynamics as will be seen below.

3. Besides those oscillatory pulse solutions, there always exists a stable steady Turing pattern or stable oscillatory Turing pattern near $\alpha=\alpha^{*}$, the mode-number of which is higher than the above oscillatory patterns and is enough to cover the whole region. This is one of the consequence that the highest mode-number of Turing pattern primarily bifurcates from the upper part of the saddle-node branch and remains as a stable steady state (or a oscillatory Turing pattern if it experiences Hopf bifurcation) for larger $\alpha$ where SRP is observed (see Section 3.1). 
4. Suppose that one takes $\alpha$ to be slightly smaller than $\alpha^{*}=0.27$, the solution orbit starting from 1-pulse shape behaves as follows: first it oscillates a couple of times with keeping one pulse shape, then splits into two pulses (duplication!) and oscillates again for a while with keeping two-pulse shape. Next one of the pulses (or both of them depending on initial data) splits again, and eventually approach the Turing pattern of 8-mode (see Fig. 3.18). It should be noted that when each multi-pulse pattern oscillates without changing its mode-number, the orbit is wondering the area in the phase space which is very close to the limiting point as in Fig. 3.17(b2). Namely the fundamental mechanism discussed in Section 3.5 is assembled in a hierarchy way, and the orbit makes a course of itineracy among oscillatory pulse solutions. Moreover, there are in general several candidates for the final destination, and hence, it is rather common to observe the subtle dependence of the final state on initial data. In fact, when we slightly change the initial data in the above simulation, the orbit goes to the oscillatory Turing pattern of 6-mode as in Fig. 3.19, although the first half of the splitting process remains as before (see Fig. 3.17(b-1)). The schematic picture depicted in Fig. 3.17 shows the essence of the transitory behavior of the self-replication. The scenario continues until the mode number does not exceed the upper limit.

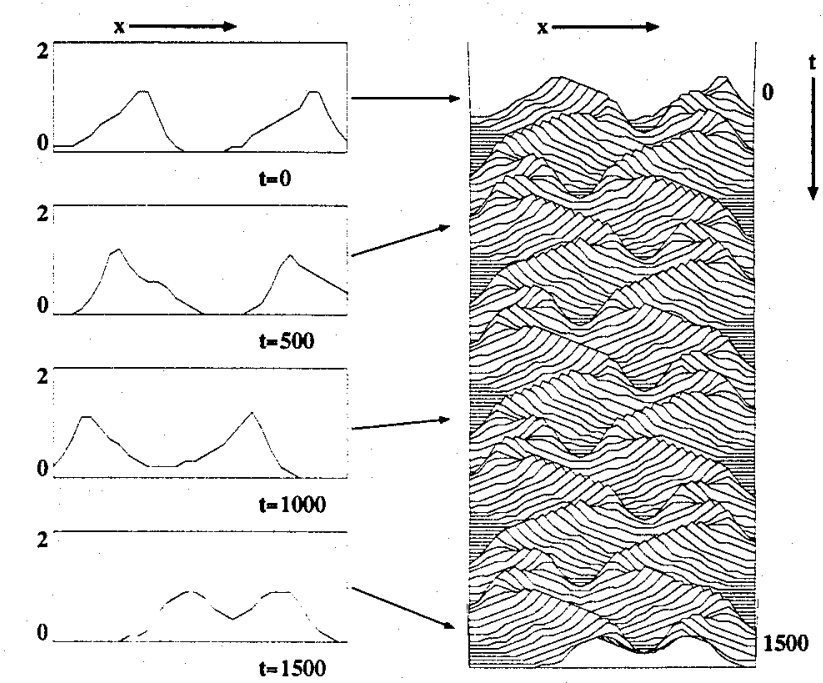

Figure 3.15: Oscillatory 2-pulses $(d=0.3, \alpha=0.272, n=32)$.

\section{Comparison with PDE model}

We compare the dynamics of the compartment model with $n=68$ with that of PDE model (1). Fig. 1.1 in Section 1 shows the replication dynamics of (1) for $\alpha=0.27169$, and similarly Fig. 4.1 for (3) with $n=68$ and $\alpha=0.27018$. There is an apparent similarity between these two simulations, although the values of $\alpha$ are slightly different. It is in 


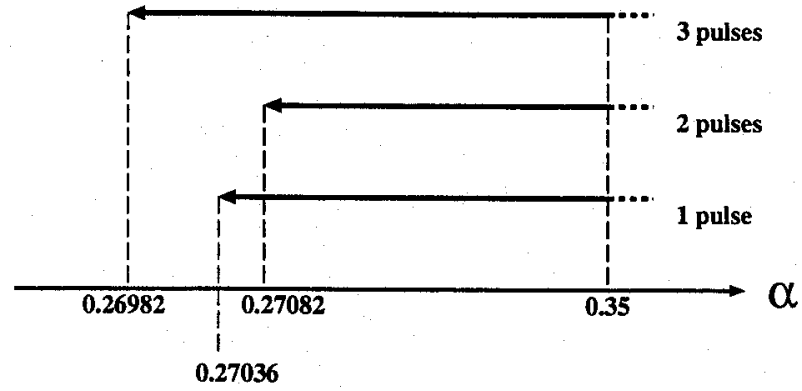

Figure 3.16: Hierarchy structure of oscillatory pulses for compartment model $(d=$ $0.3, n=32)$ : Each arrow indicates the interval where the associated pulse is stable.

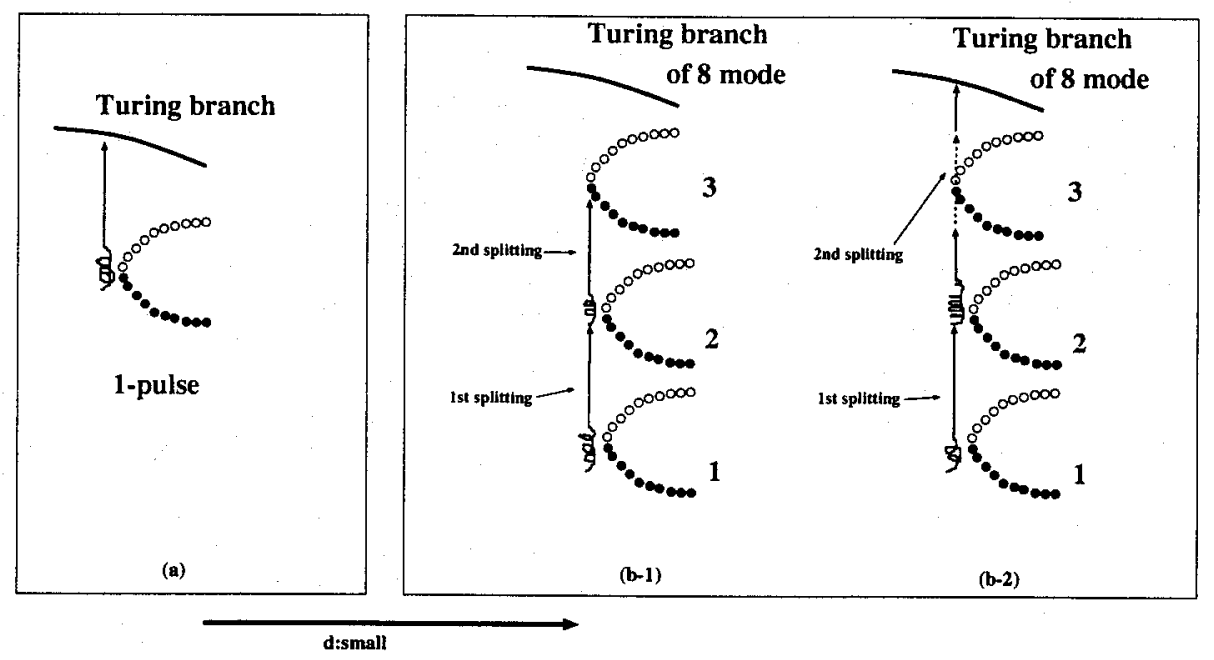

Figure 3.17: Hierarchy structure of limiting points of the oscillatory n-pulse branches. 


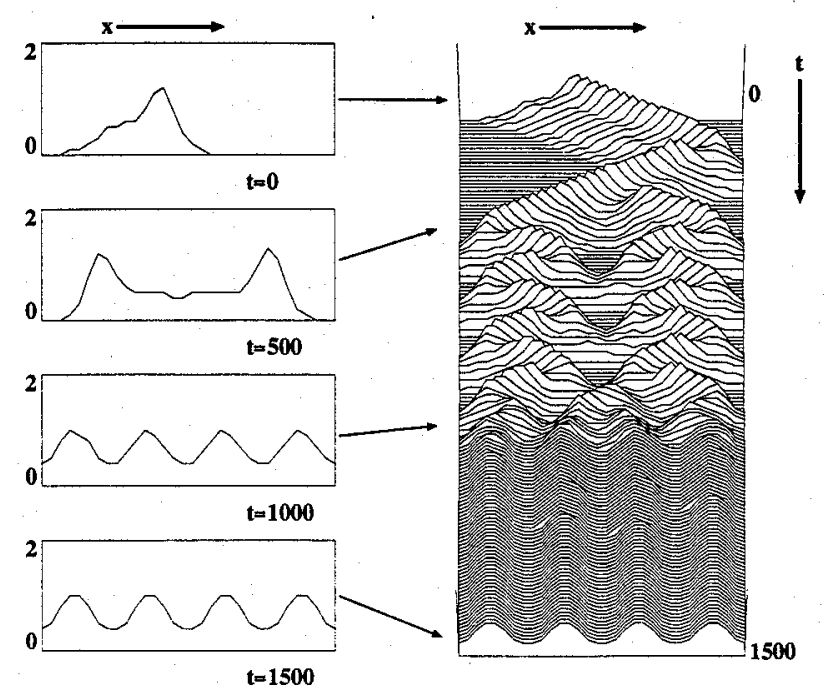

Figure 3.18: From 1-pulse to Turing pattern of 8 -mode $(d=0.3, \alpha=0.27028, n=32)$.

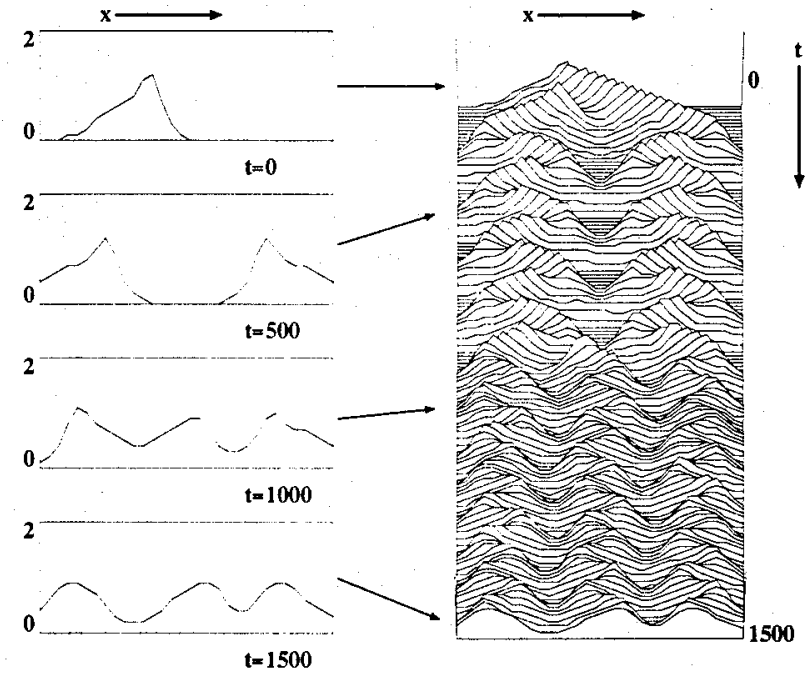

Figure 3.19: From 1-pulse to oscillatory Turing pattern of 6 -mode $(d=0.3, \alpha=$ $0.27028, n=32$ ). 
general dangerous to conclude something about the relation of the solution structure between (1) and (3) only by simulations, since (3) should be regarded to be a different model from (1). However there is a strong evidence that SRP for PDE model also stems from the same origin, namely the bifurcational structure of (1) is qualitatively similar to that of (3). For instance, the diagrams for the onset of Hopf points on Turing branches of 1-mode and 2-mode for (1) are essentially the same as in Fig. 3.3 and Fig. 3.6, and more importantly, the subcritical nature of oscillatory 1-pulse holds true for (1) as in Fig. 4.2. In general, it is quite tough and time-consuming to trace the deformation of the whole Hopf branch numerically for PDE-model, so we have not checked yet that the Hopf branch in Fig. 4.2 comes from the small Hopf barrel (this is the main reason that we adopted the compartment model). Also similar hierarchy structure of subcritical loops of oscillatory multi-pulse type is observed by checking the $\alpha$-intervals where each stable oscillatory multi-pulse exists (see Fig 4.3). Comparing this with Fig. 3.16, it is plausible that the dynamics of SRP for PDE-model comes from the same skeleton structure as (3).

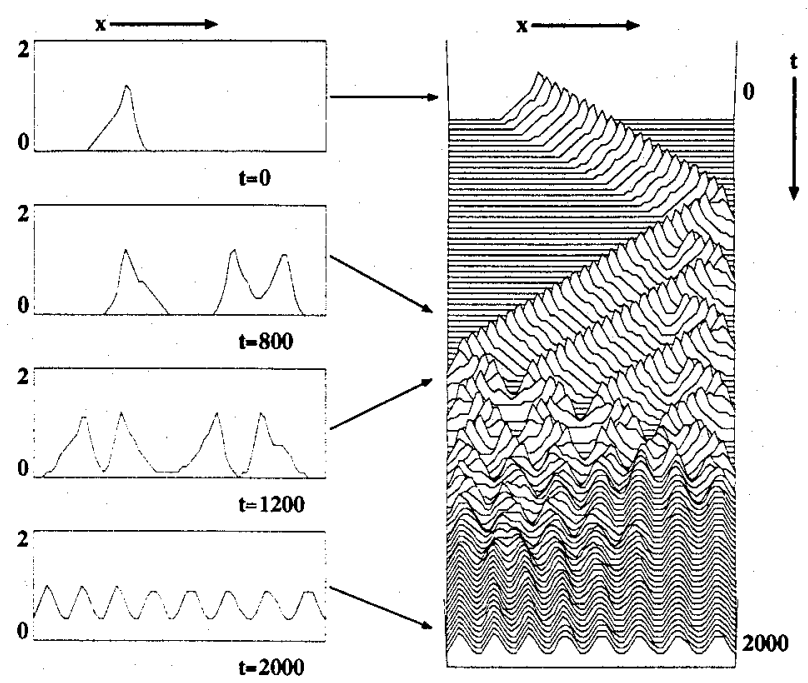

Figure 4.1: Self-replication for 68-compartment model $(d=0.3, \alpha=0.27028, n=68)$.

\section{Discussion}

We proposed a candidate for the dynamical mechanism to drive the SRP for the model system (1) on a finite interval. A hierarchy structure of limiting points of oscillatory multi-pulse branches plays a crucial role in the replication process. As we saw in Section 4 (see Fig. 3.17), in order to have SRP, the limiting points of oscillatory pulses must locate almost at the same value of $\alpha$, which can be formed only when all the parameters are tuned in an appropriately way. This is the reason for the fact that SRP was observed only in a restricted area of the parameter space. In fact, if we change $k$ or $\beta$ of (3), such a structure does not persist, and hence the exotic behavior also disappears. This is 

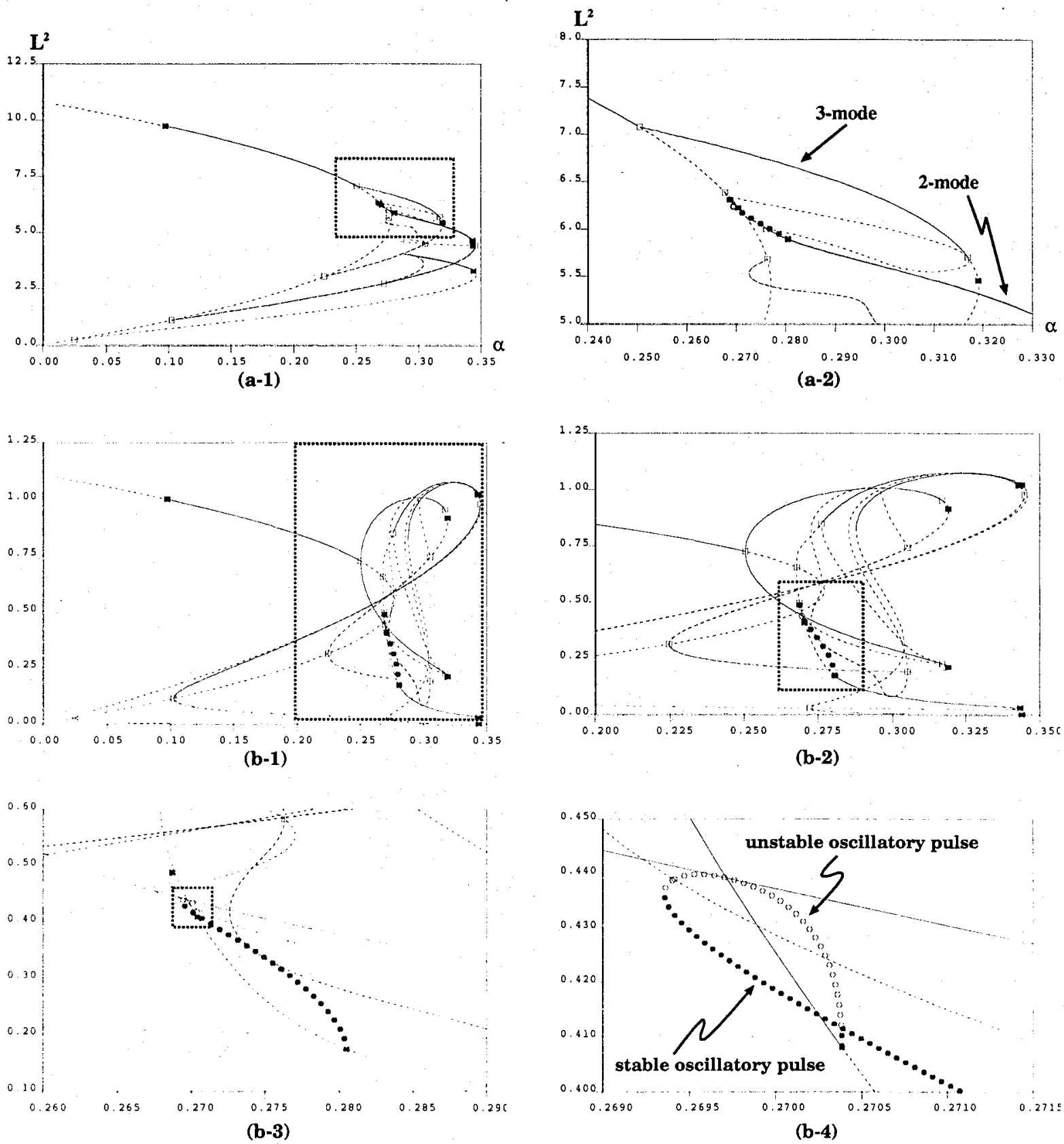

Figure 4.2: Subcriticality implies the existence of limiting point for 1-pulse branch of PDE model (system size=0.65). The norm of the vertical axis is the same as in Fig. 3.10. 


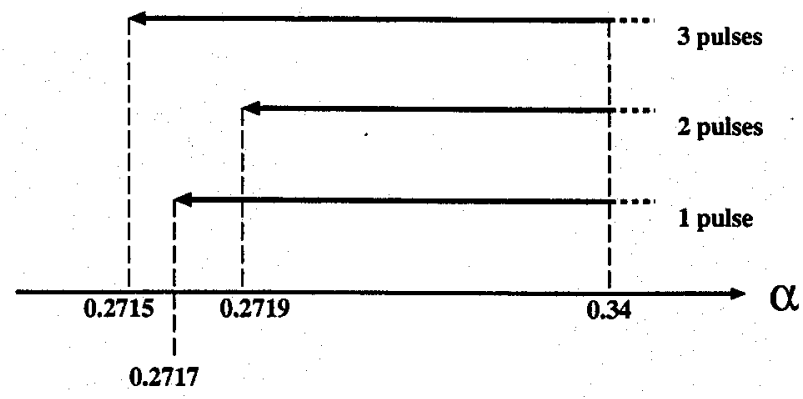

Figure 4.3: Hierarchy structure of oscillatory pulses for PDE model $\left(D_{u}=0.001, D_{v}=\right.$ 0.004 , width of interval=4).

the case also for the Gray-Scott model as in [1]. In this sense SRP is not a universally observed phenomenon for arbitrary chosen parameters. However suppose we have a stable oscillatory 1-pulse for small $d$ (or in a wider interval for a fixed $d$ ), then we have a high possibility to have SRP, because the following plausible argument seems to work to explain why the limiting points locate almost at the same value of $\alpha$. First of all, both front and tail parts of such a 1-pulse decay quickly to zero, and hence there are very few interaction among pulses when we superpose several pulses on the interval without intersection. Secondly, since the boundary condition is of Neumann type, the pulses must be robust on symmetric collision. Hence it is plausible that the solution remains as a stable oscillatory multi-pulse even after collision, when the evolution starts from such an superposed initial data. This implies that all the oscillatory multi-pulses up to some number remains stable almost on the same $\alpha$-interval. Moreover when 1-pulse becomes unstable or ceases to exist, all the other oscillatory pulses are also expected to behave in a similar way, which implies the coincidence of location of limiting points.

In 2-dimensional space we also found the self-replicating spots like Fig. 5.1 for our model system, which is quite similar to those in [1], [3], and [7]. Although we do not know the detailed structure of bifurcating localized pulses, it might be possible to know the basic mechanism if the system size is rather small. Namely, for instance, take such an domain so that only two localized spots can survive in it, then, if we start from a localized 1-spot, it may split and settle down to the two spots state, which is quite similar to the situation discussed in Section 3.4 .

Our model system (1) is different from the Gray-Scott model [10], however these two models share remarkably similar replicating dynamics. This is because both systems satisfy three conditions mentioned at the begining of Section 2.1, which, we believe, are the main ingredients to form the self-replication. The same approach employed in this paper seems to work also for the Gray-Scott model on a finite interval. The global behavior of steady branches of pulse type (i.e., standing pulses) may become important in this model. In spite of a number of open problems, our approach gives a strong evidence for the ubiquity of the proposed mechanism for SRP, and we hope that this idea can be applied to specific new problems of transient dynamics. 


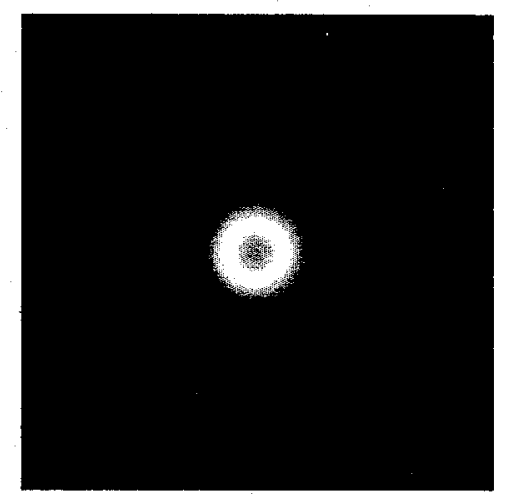

$t=200$

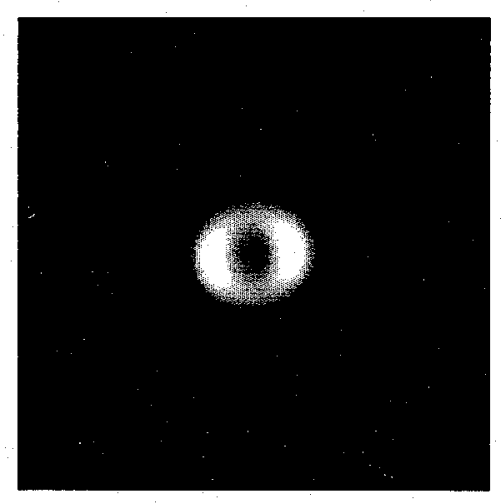

$t=400$

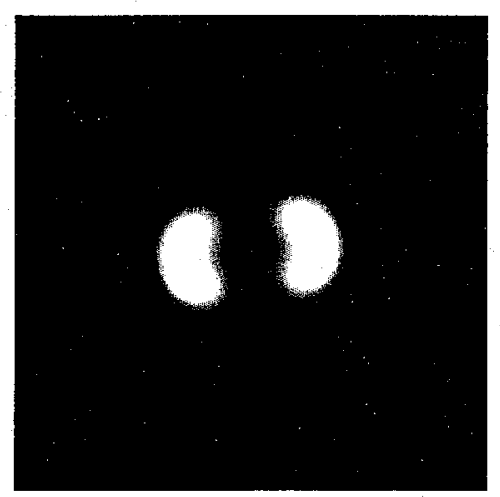

$t=800$

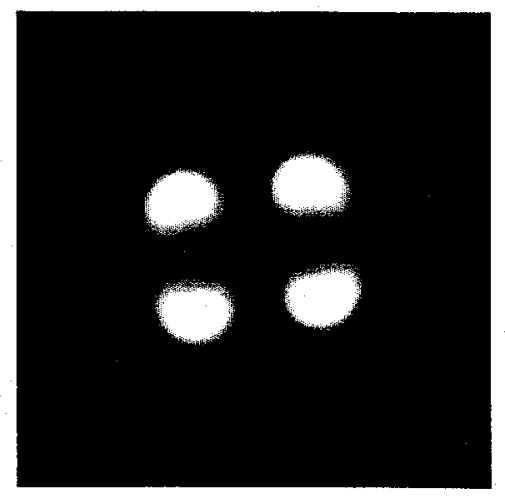

$t=1000$
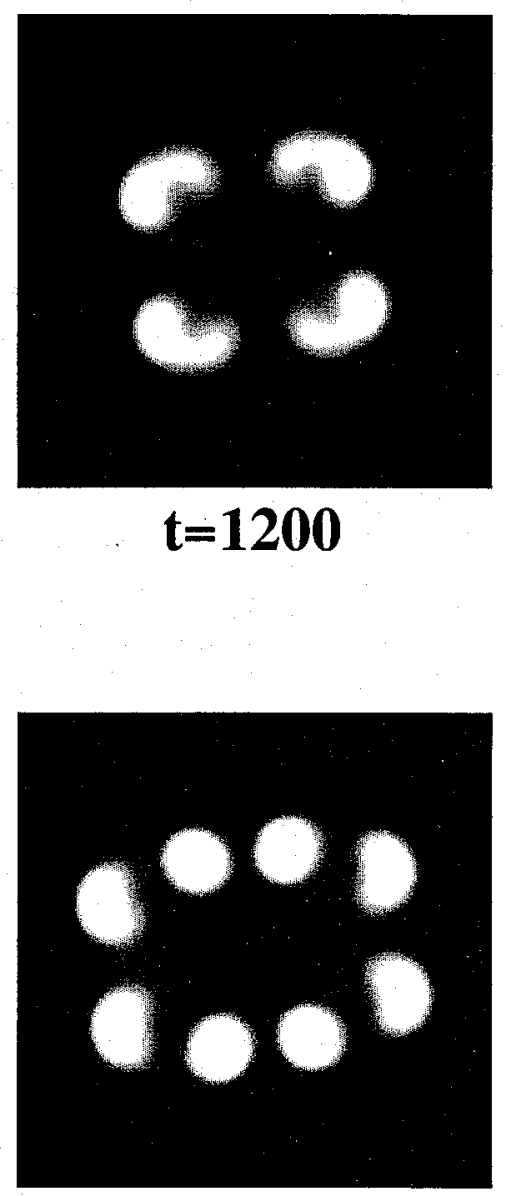

$t=1400$

Figure 5.1: Self-replicationg spots $\left(D_{u}=0.001, D_{v}=0.004, \alpha=0.275, k=1\right.$, system size $=2 \times 2$ ). 


\section{References}

[1] J.E.Pearson., Complex patterns in a simple system, Science Vol.216(1993),189-192.

[2] W.N.Reynolds,J.E.Pearson and S.Ponce-Dawson., Dynamics of self-replicating patterns in reaction diffusion systems, Physical Review Letters. Vol.72,No.17(1994),1120-1123.

[3] P.De Kepper,J.J.Perraud,B.Rudovics and E.Dulos., Experimental study of stationary turing patterns and their interaction with traveling waves in a chemical system, International Journal of Bifurcation and Chaos, Vol.4,No.5(1994) 1215-1231.

[4] K.E.Rasmussen,W.Mazin,E.Mosekilde,G.Dewel and P.Borckmans., Wave-splitting in the bistable Gray-Scott model, International Journal of Bifurcation and Chaos, Vol.6,No.6(1996) 1077-1092.

[5] V.Petrov, S.K.Scott and K.Showalter, Excitability, wave reflection, and wave splitting in a cubic autocatalysis reaction-diffusion system, Phil. Trans. Roy. Soc. Lond.A, Vol.347(1994) 631-642.

[6] A.Doelman, T.J.Kaper and P.A.Zegeling, Pattern formation in the one-dimensional Gray-Scott model, Nonlinearity, Vol.10(1997) 523-563.

[7] K.J.Lee, W.D.McCormick, J.E.Pearson and H.L.Swinney, Experimental observation of self-replicationg spots in a reaction-diffusion system, Nature, Vol.369(1994) 215218.

[8] K.J.Lee and H.L.Swinney, Lamellar structures and self-replicating spots in a reactiondiffusion system, Phys.Rev.E, Vol.51(1995) 1899-1915.

[9] E.J.Doedel, A.R.Champneys, T.F.Fairgrieve, Y.A.Kuznetsov, B.Sandstede and $\mathrm{X}$.Wang, AUTO97:Continuation and bifurcation software for ordinary differential equations (with HomCont), ftp://ftp.cs.concordia.ca/pub/doedel/auto,(1997).

[10] P.Gray and S.K.Scott, Autocatalytic reactions in the isothermal, continuous stirred tank reactor: oscillations and instabilities in the system $A+B \rightarrow 3 B, B \rightarrow C$, Chem. Eng. Sci. Vol.39(1984) 1087-1097. 